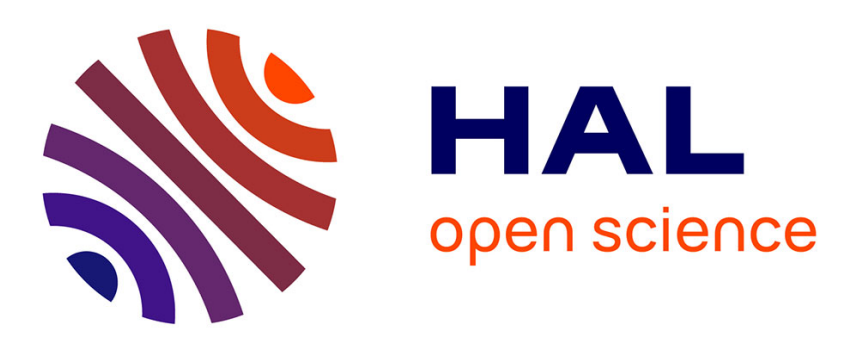

\title{
Attached cavitation in laminar separations within a transition to unsteadiness
}

Kilian Croci, Florent Ravelet, Amélie Danlos, Jean-Christophe Robinet, Luc Barast

\section{- To cite this version:}

Kilian Croci, Florent Ravelet, Amélie Danlos, Jean-Christophe Robinet, Luc Barast. Attached cavitation in laminar separations within a transition to unsteadiness. Physics of Fluids, 2019, 31 (6), pp.063605. 10.1063/1.5097924 . hal-02178972v2

\section{HAL Id: hal-02178972 \\ https://hal.science/hal-02178972v2}

Submitted on 3 Jul 2019

HAL is a multi-disciplinary open access archive for the deposit and dissemination of scientific research documents, whether they are published or not. The documents may come from teaching and research institutions in France or abroad, or from public or private research centers.
L'archive ouverte pluridisciplinaire HAL, est destinée au dépôt et à la diffusion de documents scientifiques de niveau recherche, publiés ou non, émanant des établissements d'enseignement et de recherche français ou étrangers, des laboratoires publics ou privés. 


\title{
Attached cavitation in laminar separations within a transition to unsteadiness
}

\author{
K. Croci, F. Ravelet, A. Danlos, J.-C. Robinet, and L. Barast
}

\author{
AFFILIATIONS \\ DynFluid Laboratory, ENSAM Paris, 151 Boulevard de l'Hôpital, 75013 Paris, France
}

Electronic mail: kilian.croci@gmail.com

Also at: CNAM, Paris, France.

\begin{abstract}
Attached sheet cavitation is usually observed in turbulent water flows within small laminar separation bubbles which can provide favorable conditions for inception and attachment of cavities. In the present study, viscous silicone oils are used within a small scale Venturi geometry to investigate attached cavitation into laminar separated flows for Reynolds numbers from 346 to 2188 . Numerical simulations about single phase flows are performed with steady simulations for a Reynolds number range $R e \in[50 ; 1400]$ and with unsteady simulations for $R e \in[1000 ; 2000]$. They reveal the emergence of two large laminar boundary layer separations downstream of the Venturi throat in addition to low pressure zones which can possibly induce both degassing or cavitation features. Experiments are performed with high-speed photography, and several multiphase dynamics are observed in these viscous flows, which are considered as quasisteady flows at low Reynolds numbers $R e \leq 1400$. Degassing phenomenon with air bubble recirculation has been first observed at pressures far above liquid vapor pressure whereas typical attached cavities have been identified for low pressure conditions as "band" and "tadpole" cavities into the different separations of the laminar flows. For higher Reynolds numbers, a flow regime transition can be noticed in the wake of well-developed gas structures, characterized by wake instabilities, causing vortex cavitation above a critical Reynolds number associated with the bubble width $R e_{c}^{b} \simeq 616$. This regime transition can possibly occur either quasicontinuously in the wake of an attached "band" vapor cavity or intermittently behind a recirculating air bubble generated with degassing. This last phenomenon is associated in our study to classical "patch" cavitation.
\end{abstract}

\section{INTRODUCTION}

Attached cavitation is generally defined as the establishment of a stable bubble, or cavity, in a particular location of a flow such as boundary layer separations usually induced by high pressure gradients $^{1}$ with, for instance, laminar separation bubbles ${ }^{2}$ into turbulent flows. Arakeri ${ }^{3}$ and Franc and Michel ${ }^{4}$ introduced this kind of cavitation, usually associated with "sheet" cavitation, in turbulent flows within a laminar separation bubble in which cavities can emerge and develop without being carried away by the incoming flow. Attached cavities have thus been observed on smooth axisymmetric bodies by Parkin and Kermeen ${ }^{5}$ and Arakeri, ${ }^{6}$ on propellers blades by Kuiper, on foils by Guennoun, and around a sphere by Brandner et al. ${ }^{9}$ This stable attached sheet cavitation can possibly transition to partial "cloud" cavitation ${ }^{10}$ where classical re-entrant jet ${ }^{11}$ and bubbly shock wave phenomena ${ }^{12}$ can occur periodically. Different types of attached cavities can be observed in that sort of flow such as attached band "finger" cavities, ${ }^{8}$ which are generated in the separation layer when the vapor pressure is reached at some point, or even patch cavitation $^{13}$ which emerges when a large bubble interacts with the laminar separation bubble leading to instabilities that can induce vaporization. These attached cavities are reviewed in detail by Van Rijsbergen. ${ }^{14}$

Furthermore, another phenomenon can have an important impact in such types of cavitating flows, the degassing. BriançonMarjollet, Franc, and Michel ${ }^{15}$ thus investigated the effects of air bubble injection on vapor cavities attached on a hydrofoil. They observed that the air bubbles appeared to deeply change the flow topology, modifying quite frequently the adverse pressure gradients leading to a destabilization of the laminar separation bubble in which stable vapor cavities were initially attached. Consequently, stable attached cavitation transforms with bubble injection into traveling bubble cavitation which is characterized by nuclei growth in low pressure regions and collapse within higher pressures. ${ }^{16}$ 
Venning et al. ${ }^{17}$ added that an important population of free gas nuclei can even impact developed partial cloud cavitation shedding mechanisms. More generally, the influence of free stream nuclei ${ }^{18,19}$ in addition to noncondensable dissolved gas ${ }^{20}$ on cavitation inception has already been evidenced and a hysteresis between inception and desinence is classically observed depending on these two parameters. Dissolved air concentrations and thus air bubbles' population can be particularly important in oil viscous flows, ${ }^{21}$ with a typical Bunsen coefficient ${ }^{22}$ from 7 to $12 \mathrm{Vol} . \%{ }^{23}$ leading to similar cavitation inception hysteresis ${ }^{24}$ than those classically observed in turbulent water flows. The inception of cavitation is commonly associated in the literature ${ }^{25}$ to a nuclei which fills with vapor when it experiences a pressure drop below the liquid vapor pressure. Washio, Takahashi, and Yoshimori, ${ }^{26}$ Washio et al., ${ }^{27}$ and Washio, Kikui, and Takahashi ${ }^{28}$ observed vaporous cavitation inception in viscous mineral oil separated flows and proposed another inception mechanism which does not depend on the presence of a nuclei. According to their studies, vapor cavities can emerge close to a separation point on the wall where the liquid undergoes high tensions which can, when the threshold is exceeded, induce a microscopic rift between the liquid and the wall leading to the emergence of a cavity.

In summary, a separated viscous flow should present favorable conditions for the emergence of attached cavitation in addition to an important degassing, which could possibly interact with both vapor cavities and flow separations. In a previous work, Croci et al. $^{29}$ observed the formation of attached cavities, supposedly filled with air regarding the high pressure measured, generated with the interaction of degassing with a large separated laminar viscous flow.

In the present study, this investigation is extended to cavitation inception and development of partial cavitation into viscous separated laminar flows within a Venturi geometry. In these viscous flows, shedding mechanisms as re-entrant jets or bubbly shock waves, usually observed in turbulent water flows, do not seem to develop contrary to attached cavitation. The viscous silicon oils used, with a high dissolved air content (the Bunsen coefficient was about 19 Vol. $\%$ at $25^{\circ} \mathrm{C}$ for $47 \mathrm{~V} 50$ oil), can also present important degassing dynamics. The attached cavities, both resulting from degassing (air) or cavitation (oil vapor), are investigated experimentally with high speed cameras exploring increasing inlet Reynolds numbers from roughly 350 to 2200 as a function of the flow cavitation number. A typical map presenting both cavitation and degassing flow conditions is sketched in Fig. 1 highlighting a flow transition to unsteadiness which can occur, for this range of Reynolds numbers, depending on the multiphase dynamics present.

In cavitating flows, instabilities can induce important pressure fluctuations which can modify attached cavitation inception and development. ${ }^{30}$ These fluctuations might also impact degassing dynamics as illustrated in Fig. 1. Consequently, the final part of this study will focus on the particular flow dynamics that might be due to instabilities and indicate a flow regime transition to unsteadiness.

After introducing the experimental setup and protocol in Sec. II, the flow topology is highlighted in Sec. III, first numerically with both steady $(R e \in[50 ; 1400])$ and unsteady $(R e \in[1000$; 2000]) simulations and second with an experimental observation

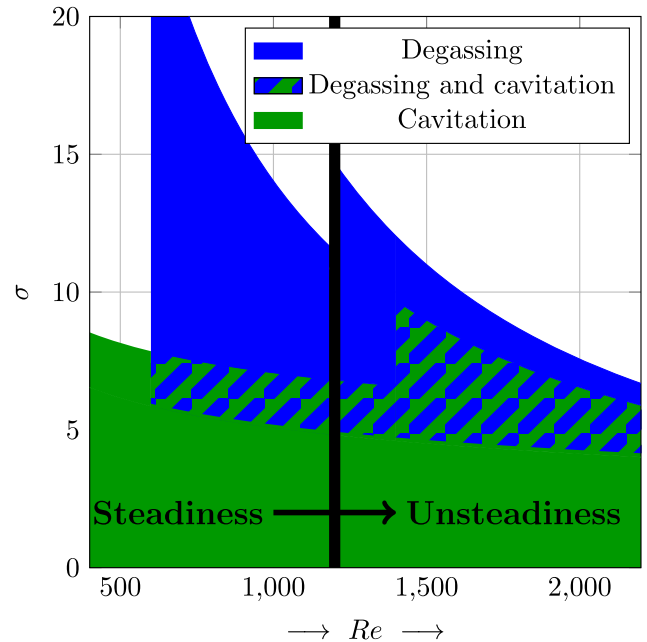

FIG. 1. Typical multiphase phenomena map (based on the experimental outcomes developed in Secs. IV and V) that occur in silicon oil flows sketched depending on the cavitation and the Reynolds numbers. Degassing and cavitation dynamics can importantly modify the flow topology and induce a flow transition to unsteadiness.

of bubble recirculation indicating a flow separation $(R e \simeq 652$ and $\sigma \simeq 17.9$ in $47 \mathrm{~V} 100$ silicone oil). $47 \mathrm{~V} 100$ viscous oil flows presenting low Reynolds numbers $(R e \leq 1201)$ are then investigated experimentally. These flows, which are considered as quasisteady flows, present different attached cavities which are fully described in Sec. IV. In a second part, the destabilization of the previous steady laminar flow is investigated using a $47 \mathrm{~V} 50$ less viscous silicon oil in order to explore higher Reynolds number conditions until $R e \simeq 2188$. In Sec. V, flow unsteadiness is investigated revealing two main unsteady flow dynamics:

- "Patch" cavitation (Sec. V A) which emerges intermittently.

- Wake vortex cavitation (Sec. V B) which occurs at high frequency.

Finally, the study conclusions are developed in Sec. VI presenting equally some future works.

\section{EXPERIMENTAL OVERVIEW}

\section{A. Cavitation test-loop and protocol}

The experiments were conducted in a test-loop, presented in Fig. 2, of the DynFluid laboratory facilities especially designed to limit pressure loss and to visualize cavitation. The loop is composed of a Pollard MPLN 142 centrifugal pump (1) which impels silicon oil in a pipe system presenting 40 and $50 \mathrm{~mm}$ inner diameters to a $100 \mathrm{l}$ tank (2). The oil tank, which is equipped with a temperature sensor ThermoEst PT100 (3), is connected to air supply or to a vacuum pump (4) to set the static pressure in the test section when operating. The oil goes then up to an ultrasonic flowmeter KHRONE Optisonic 3400 (5) placed 1.5 meters upstream of the test section (7) in which the pressure is monitored with two pressure sensors (6) located, respectively, at the inlet and the outlet sections. 


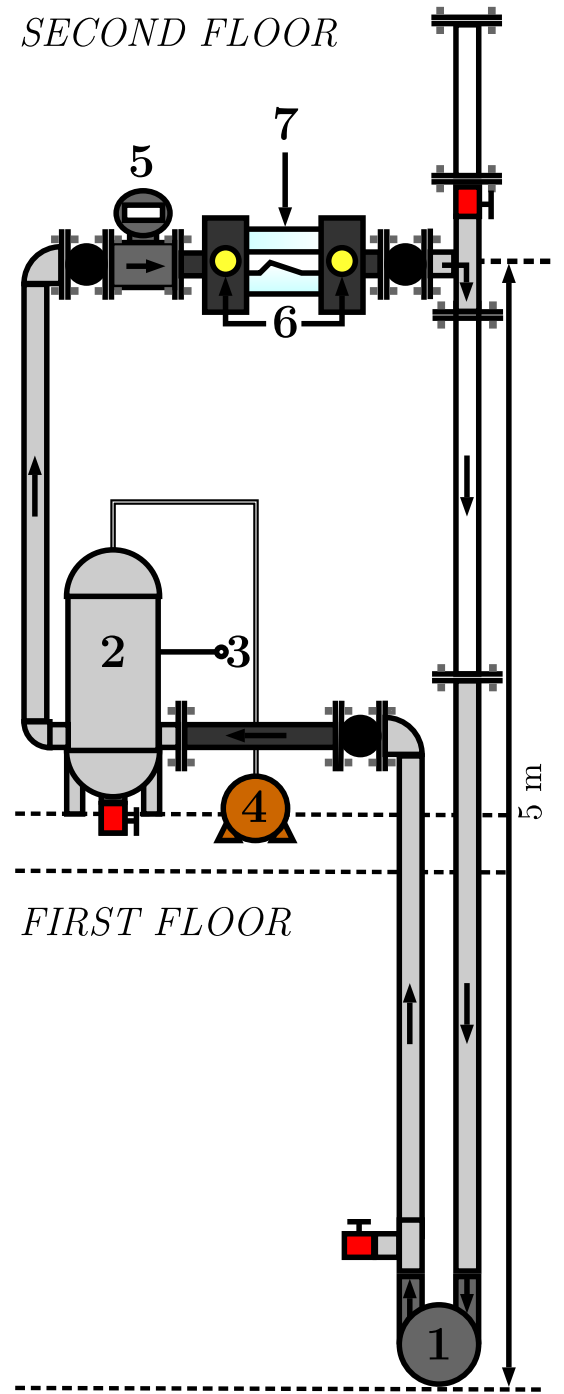

FIG. 2. Sketch of the test loop with (1) the pump, (2) the oil tank, (3) the temperature sensor, (4) the compressor or vacuum pump, (5) the ultrasonic flowmeter, (6) the pressure sensors, and (6) the operating test section detailed in Fig. 3. Reprinted with permission from K. Croci, "Étude expérimentale des écoulements multiphasiques dans une couche limite laminaire decollée," Ph.D. thesis, Arts et Métiers Paris, France, 2018.
The test section, schematized in Fig. 3, consists of a Venturi geometry with convergent/divergent angles of $18^{\circ}$ and $8^{\circ}$, respectively, similarly to a previous study of Danlos et al. ${ }^{31}$ It presents a $20 \times 10 \mathrm{~mm}^{2}$ inlet (or outlet) section and a $10 \times 10 \mathrm{~mm}^{2}$ square section at the throat. The test section is all made of transparent plexiglass in order to permit both side and top visualizations. Two round to rectangle contraction nozzles with an area ratio of 6.3 are placed on each side of the test section to connect to the pipe system.

All experiments are realized following a similar protocol in order to investigate separated viscous flows. The oil in the setup is first kept saturated at the atmospheric pressure, circulating at this pressure at a slow velocity during several hours, and experiments are carried out progressively decreasing the pressure in the system in order to always get the oil at supersaturation, allowing the driven diffusion process described by Groß and Pelz. ${ }^{32}$ The measurements are operated, for stabilized temperatures taken in a range $15.5<T<27.1^{\circ} \mathrm{C}$, at least $5 \mathrm{~min}$ after modifying the flow parameters to steady the flow. All inception measurements are reproduced three times at fixed velocities, slowly decreasing the pressure in the test section within a waiting time about 10 min between each point to capture the phenomenon emergence in stable flow conditions. The inlet and outlet test section pressures, named $P_{\text {in }}$ and $P_{\text {out }}$, are measured with pressure taps located at $103 \mathrm{~mm}$ upstream and $201 \mathrm{~mm}$ downstream from the Venturi throat, respectively (Fig. 3). The pressure loss $\Delta P$, that will be used in Sec. III A, represents the difference between $P_{\text {in }}$ and $P_{\text {out }}$. The inlet velocity $V_{\text {in }}$ is estimated from flowrate measurements applied to the inlet test section. In these experiments, the inlet velocity ranges between 1.5 and $5.9 \mathrm{~ms}^{-1}$ and the associated inlet Reynolds number, defined as $R e=U_{\text {in }} h_{\text {in }} / v$ (with $v$ the kinematic viscosity of the liquid), is explored for $346<R e<2188$. The cavitation number is equally defined at the inlet section as $\sigma=2\left(P_{\text {in }}-P_{v}\right) / \rho U_{i n}^{2}$, where $\rho$ and $P_{v}$ are the density and the vapor pressure of the liquid, respectively. All lengths are normalized with the test section width $w=10 \mathrm{~mm}$ such as $X^{\star}=X / w$.

\section{B. Study parameters and flow visualization}

The two viscous oils used in this study are given as follows:

- The 47 V 50 silicon oil (at temperature $T=25^{\circ} \mathrm{C}$, density $\rho=959 \mathrm{~kg} \mathrm{~m}^{-3}$, kinematic viscosity $v=49.4 \mathrm{~mm}^{2} \mathrm{~s}^{-1}$, and surface tension $S=20.7 \mathrm{mN} \mathrm{m}^{-1}$ ), already studied by Duhar and $\operatorname{Colin}^{33}$ to investigate bubble detachment in shear flows.

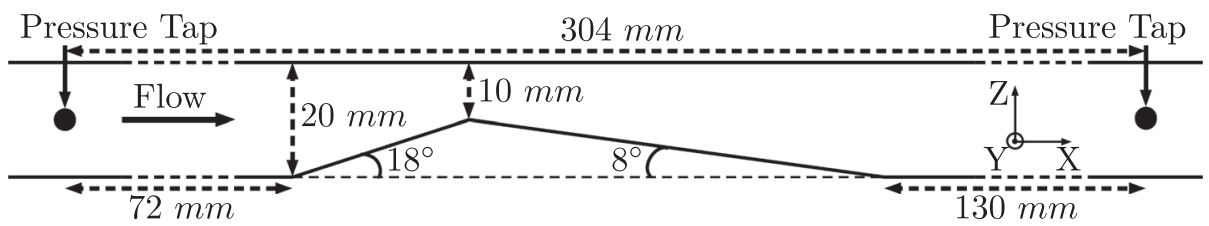

FIG. 3. Sketch of the Venturi geometry. The width of the test section is $w=10 \mathrm{~mm}$ and its height at the inlet section is $h_{\text {in }}=20 \mathrm{~mm}$. Thereafter, the axes origin is positioned at the Venturi throat edge in the middle of test section along the width. Reprinted with permission from K. Croci, "Étude expérimentale des écoulements multiphasiques dans une couche limite laminaire decollée," Ph.D. thesis, Arts et Métiers Paris, France, 2018. 

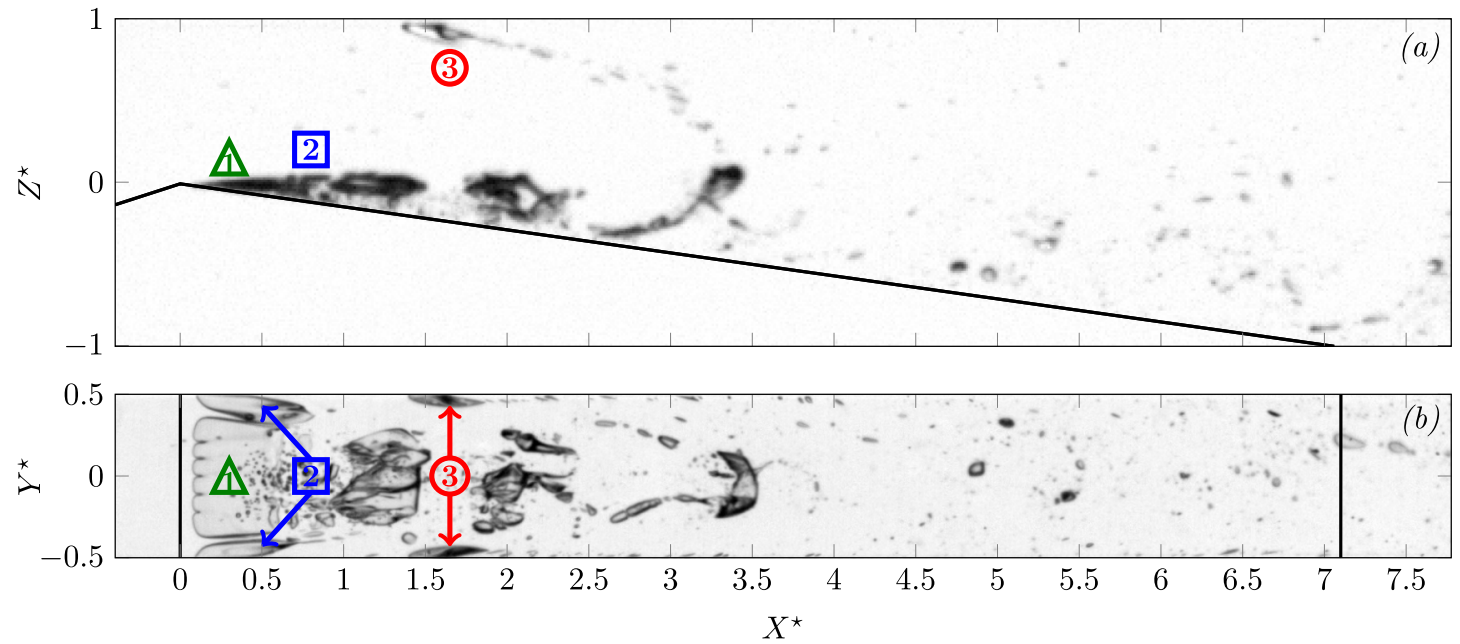

FIG. 4. Side (a) and top (b) views of typical transitioning flow, with attached cavities and vaporization of a hairpin vortex, captured at $R e=2185$ and $\sigma=4.53$. The flow is from left to right. The two black lines positioned at $X^{\star}=0$ and $X^{\star}=7.1$ indicate the throat and the end of the Venturi slope, respectively. The different attached cavities usually observed in laminar separated flows, namely, the "band" cavity, the "tadpole" cavities, and the secondary "tadpole" bubbles, are associated with symbols (green open triangle, blue open square, and red open circle) and numbered from 1 to 3 , respectively. Reprinted with permission from K. Croci, "Étude expérimentale des écoulements multiphasiques dans une couche limite laminaire decollée," Ph.D. thesis, Arts et Métiers Paris, France, 2018.

- The $47 \mathrm{~V} 100$ silicon oil which at temperature $T=25^{\circ} \mathrm{C}$ has a density $\rho=963 \mathrm{~kg} \mathrm{~m}^{-3}$, a kinematic viscosity $v=101.2 \mathrm{~mm}^{2} \mathrm{~s}^{-1}$, and a surface tension $S=20.9 \mathrm{mN} \mathrm{m}^{-1}$.

Both silicone oils, which present a good transparency for visualization, vaporize at a really low vapor pressure $P_{v} \simeq 1 \mathrm{~Pa}$. Oil flow capillary numbers, defined as $C a=\rho v V_{\text {in }} / S$, present high values over $10^{2}$ revealing the negligibility of capillary forces compared to viscous forces in our flows. The $47 \mathrm{~V} 100$ oil is first used for the measurements developed in Sec. IV, whereas the images depicted in Sec. V are for $47 \mathrm{~V} 50$ oil flows, which is less viscous.

Cavitating flows are captured with two Optronis CR1000 $\times 3$ high-speed cameras placed on the top and the side of the test section, respectively. The images are captured with a frame rate of 1500 frames/s with a resolution of $1280 \times 768$ pixels $^{2}$ for $2 \mathrm{~s}$. The resulting photographs are then normalized with a background image (without cavitation). The flow is continuously illuminated from the backside with a homogeneous white LED backlight Phlox and, consequently, the interface between the liquid and vapor appears in black in the images. Typical side and top views of a cavitating flow in a transitioning flow regime (cf. Sec. V) are depicted in Fig. 4.

All the main study parameters are presented in Table I with their associated uncertainties. In Secs. III-V, two main parameters will be used to characterize each flow: the Reynolds and the cavitation numbers.

\section{LAMINAR FLOW TOPOLOGY}

\section{A. Numerical laminar flow}

Numerical simulations of the single-phase flow are performed in order to study both the topology of the flow and the order of magnitude of the absolute pressure encountered in the experiment, as a function of the Reynolds number. The computational domain

TABLE I. Flow parameters, ranges, and estimated uncertainties for silicone oils $47 \mathrm{~V} 50$ and $47 \mathrm{~V} 100$.

\begin{tabular}{llcccc}
\hline \hline Symbol & \multicolumn{1}{c}{ Parameters } & Range $(47 \mathrm{~V} \mathrm{50)}$ & Range $(47 \mathrm{~V} \mathrm{100)}$ & Unit & Uncertainty (\%) \\
\hline $\mathrm{Ca}$ & Capillary number & {$[420 ; 1240]$} & {$[1041 ; 2611]$} & $\ldots$ & \pm 3 \\
$P_{\text {in }}$ & Inlet pressure & {$[355 ; 1100]$} & {$[617 ; 1531]$} & $\mathrm{mbar}$ & $<1$ \\
$P_{\text {out }}$ & Outlet pressure & {$[117 ; 753]$} & {$[155 ; 1202]$} & $\mathrm{mbar}$ & $<1$ \\
$R e$ & Inlet Reynolds number & {$[541 ; 2188]$} & {$[346 ; 1201]$} & $\ldots$ & \pm 3 \\
$T$ & Operating temperature & {$[15.5 ; 26.1]$} & {$[18.4 ; 27.1]$} & ${ }^{\circ} \mathrm{C}$ & $<1$ \\
$V_{\text {in }}$ & Inlet velocity & {$[1.51 ; 5.56]$} & {$[1.95 ; 5.86]$} & $\mathrm{ms}^{-1}$ & $<1$ \\
$\sigma$ & Inlet cavitation number & {$[4.53 ; 73.0]$} & {$[5.73 ; 60.0]$} & $\ldots$ & \pm 2 \\
$\rho$ & Oil density & {$[958 ; 964]$} & {$[961 ; 970]$} & $\mathrm{kg} \mathrm{m}^{-3}$ & $<1$ \\
$v$ & Oil kinematic viscosity & {$[48.2 ; 59.7]$} & {$[96.9 ; 115]$} & $c S t$ & \pm 2 \\
\hline \hline
\end{tabular}


reproduces the experimental geometry that is shown in Fig. 3. It is extended by extrusion in the $X$ direction on a distance $10 w$ upstream of the inlet of the test section and $20 \mathrm{w}$ downstream of the outlet of the test section. The computational domain is meshed with $1.13 \times 10^{6}$ parallelepipedic cells with 51 points in the crossstream directions and a near-wall cell size of $5 \times 10^{-3} \mathrm{w}$. A grid convergence test has been performed at $R e=1500$. The boundary conditions are a uniform inlet velocity and a uniform static pressure outlet. The Computational Fluid Dynamics code that is used is StarCCM+. The equations that are solved are the incompressible Navier-Stokes equations with a constant density and viscosity. No turbulence model is used. The scheme of the spatial discretization is second order upwind and the velocity pressure coupling uses a co-located variable arrangement and a Rhie-andChow-type pressure-velocity coupling combined with a SIMPLEtype algorithm. Steady simulations have been performed in the range $R e \in[50 ; 1400]$, and unsteady simulations with a secondorder Euler implicit method have been performed in the range $R e \in[1000 ; 2000]$.

The dimensionless pressure difference across the test section is plotted as a function of the Reynolds number in Fig. 5 and is compared to experiments performed under single-phase flow conditions. The pressure loss coefficient is defined as $K_{p}=2\left(P_{\text {in }}-P_{\text {out }}\right) / \rho U_{\text {in }}^{2}$. The two curves fairly collapse, which is a clue in favor of the quality of the simulations. Moreover, one can notice that the scaling of the pressure loss coefficient with the Reynolds number roughly corresponds to a $R e^{-1}$ power-law at a very low Reynolds number $(R e \in[50 ; 150])$ which is consistent with the laminar flow. The local scaling exponent then gradually increases from $\kappa \simeq-0.94$ at $R e=200$ to $\kappa \simeq-0.48$ at $R e=1000$ and $\kappa \simeq-0.25$ at $R e=1400$ (see the inset in Fig. 5). There is thus no evidence for a bifurcation in the flow but rather for a smooth and gradual change in the topology of the flow with the increase in the Reynolds number. This corresponds to the development of a separation bubble downstream of the venturi throat as described hereafter.

In the range of Reynolds numbers investigated $(\operatorname{Re} \in[0 ; 2000])$, two distinct flow separations, illustrated in Fig. 6 with velocity isocontours, can emerge:

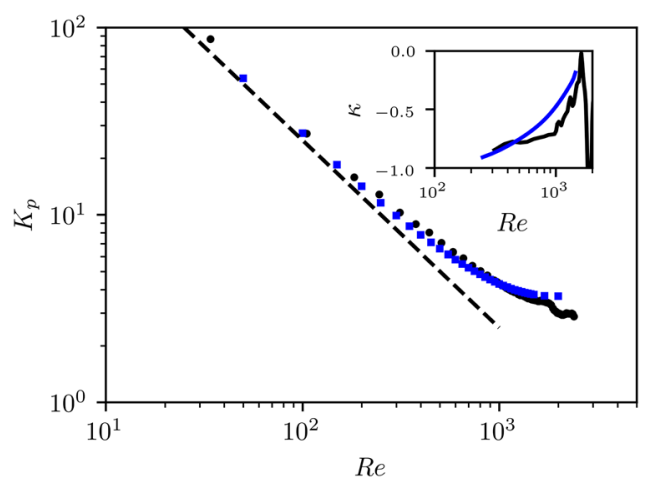

FIG. 5. Pressure loss coefficient $K_{p}$ as a function of the Reynolds number $R e$. The black bullets stand for experiments in single-phase flow and the blue squares stand for the numerical simulations. The dashed line is a line of equation $K_{p} \propto R e^{-1}$. Inset: local scaling exponent $\kappa$ such that $K_{p} \propto R e^{\kappa}$.

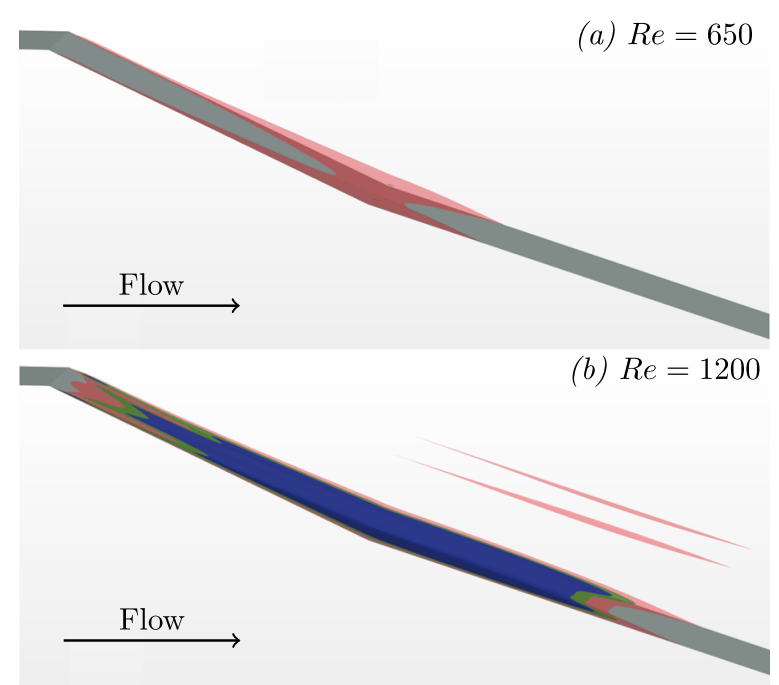

FIG. 6. $X$-axis velocity isocontours highlighting the flow separations in flows presenting Reynolds numbers (a) $R e=650\left(V_{i n}=3.25 \mathrm{~ms}^{-1}\right)$ and (b) $R e=1200$ $\left(V_{\text {in }}=6 \mathrm{~ms}^{-1}\right)$. Red, green, and blue regions correspond to $X$-axis velocity $V_{x}=-10^{-5},-0.2$, and $-0.4 \mathrm{~ms}^{-1}$, respectively.

- A main flow separation, which occurs starting from a Reynolds number $R e \simeq 350$, develops in the wake of the Venturi geometry along the divergent slope corresponding to the position $X^{\star}>0$ and $Z^{\star}<0$, first along each lateral wall $\left(Y^{\star} \simeq \pm 0.5\right)$ and on all along the Venturi width for $R e \geq 650$ as depicted in Fig. 6(a).

- A secondary laminar boundary layer separation emerges at a Reynolds number $R e \simeq 1100$ at the upper wall of the test section $\left(X^{\star}>0\right.$ and $\left.Z^{\star} \simeq 1\right)$. This flow separation, shown in Fig. 6(b) for the flow Reynolds number $R e=1200$, can be associate to that classically observed downstream of a backward-facing step by Armaly et al. ${ }^{34}$

The different flow separations illustrated in Fig. 6 for (a) $R e=650$ and (b) $R e=1200$ present counterflow streams inducing a recirculation dynamics that will be developed experimentally in Sec. III B. Figure 7 presents the X-axis velocity (b) and pressure (c) profiles that are extracted along a line situated $0.5 \mathrm{~mm}$ [i.e., $5 h_{\text {in }} / 100$ above the bottom wall and at $Y^{\star}=0$ (a)]. The velocity profiles for the Reynolds numbers $R e=650$ and $R e=1200$, normalized with the inlet velocity $V_{\text {in }}$, reveal the flow acceleration due to the section reduction at the Venturi throat and the flow separation on the Venturi divergent slope, which increases with the Reynolds number. First, upstream of the venturi throat (for $X^{\star} \leq-3$ ), the dimensionless velocities are quite low owing to the presence of a boundary layer. They are also different for the two different Reynolds numbers which illustrates the continuous change in flow topology with the Reynolds number. In both cases, the maximum dimensionless velocity is reached at the throat $\left(X^{\star}=0\right)$ and is moreover surprisingly large for $R e=1200$. The velocity profile then decreases very fast, with $V_{x} \leq 0.2 V_{\text {in }}$ for $X^{\star} \geq 1$. The streamwise velocity along this line that lays in the plan $Y^{\star}=0$ becomes negative only very close to the end of the venturi slope $\left(X^{\star}=7.1\right)$ at $R e=650$, whereas a large 

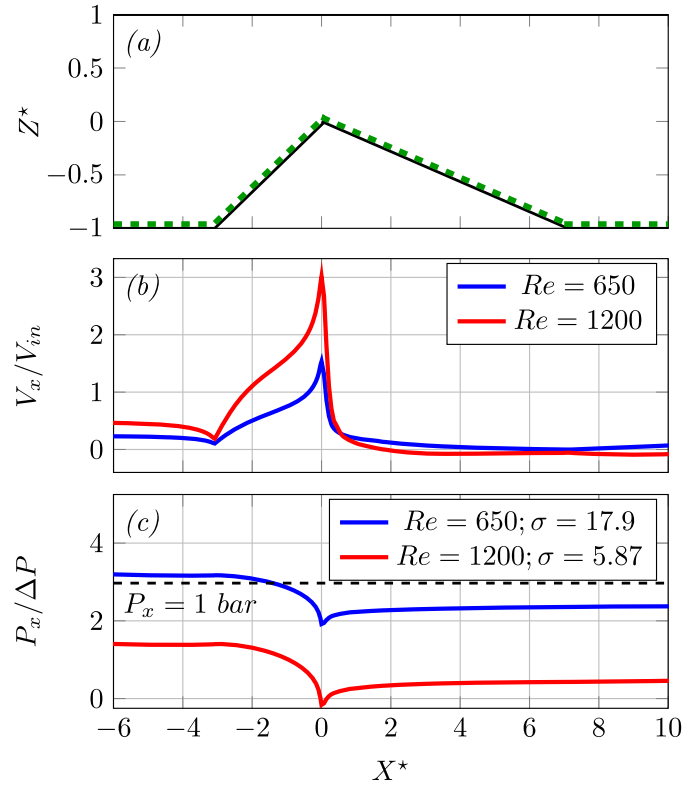

FIG. 7. Venturi geometry (a), X-axis velocity $V_{x}$ (b), and pressure $P_{x}$ (c) profiles measured along the Venturi geometry wall at $Y^{\star}=0$ for Reynolds numbers $R e=650$ and 1200. $V_{x}$ and $P_{x}$ are normalized with the inlet velocity $V_{i n}$ and the pressure loss $\Delta P$, respectively. The dashed green line in (a) reveals the measure line, located $0.5 \mathrm{~mm}$ above the bottom wall at $Y^{\star}=0$, from which pressure and velocity profiles are extracted. The black dashed line plotted in (c) corresponds to the condition $P_{x}=1$ bar, indicating the air saturating level used in our experiments.

area with negative streamwise velocities is visible at $R e=1200$, for $X^{\star} \in[1.8 ; 11.8]$. This is consistent with the isocontours shown in Fig. 6.

These different laminar flow separations can provide favorable conditions to the attachment of gas cavities resulting, for instance, from cavitation. As illustrated in Fig. 7(c) with the pressure profile along the Venturi geometry, normalized with the pressure loss $\Delta P$, the flow acceleration at the throat induces a low pressure region located in the vicinity of the Venturi throat $\left(X^{\star}=Y^{\star}=Z^{\star} \simeq 0\right)$. Note that these profiles depend on both the Reynolds number and the cavitation number, with the dimensionless value of the inlet pressure being $\sigma / K_{p}$. These profiles are shown for two flow conditions that will be investigated experimentally in Secs. III B and IV: $R e=650$ and $\sigma=17.9$ on the one hand, and $R e=1201$ and $\sigma=5.87$ on the other hand. In the first case ( $R e=650$; $\sigma=17.9$ ), we can notice that the absolute pressure is greater than the air saturation pressure upstream of the throat and that downstream of the throat, at least along this peculiar line, degassing may occur. In the second case $(R e=1201 ; \sigma=5.87)$, the absolute pressure appears to reach negative values close to the Venturi throat, implying the possible vaporization of liquid oil in this part of the flow.

We thus estimate numerically in Fig. 8 the value of the critical cavitation number that leads to a minimal absolute pressure of $1.3 \mathrm{~Pa}$ at this particular position as a function of the Reynolds number.

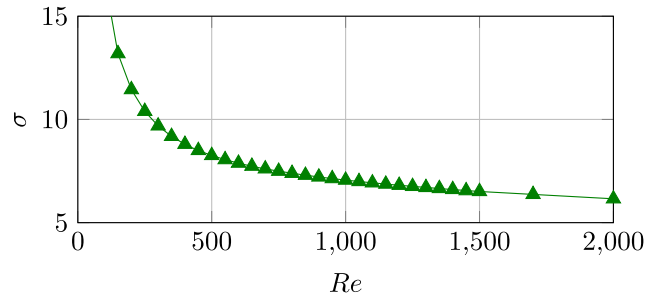

FIG. 8. Estimation of cavitation emergence into numerical steady $(R e \in[0 ; 1400])$ and $(R e \in[1400 ; 2000])$ unsteady flows depending on Reynolds and cavitation numbers.

For flow conditions located under the curve presented in Fig. 8, the Venturi geometry would generate enough depression near the throat to induce the oil to vaporize. In Secs. III B-V B, this curve will be used in order to analyze experimental laminar flows and more particularly cavitation inception and flow stability.

\section{B. Flow separation and recirculation}

In our multiphase experiments, the flow separations are characterized by bubbly recirculating processes. This dynamic is illustrated in Fig. 9 for $R e=652$ and $\sigma=17.9$. For this particular flow conditions, the one-phase flow topology has been highlighted in Figs. 6(a) and $6(\mathrm{~b})$. In these figures, we can notice that the flow separation, located at the end of the Venturi slope $\left(X^{\star}=7.1\right)$, is developed on all the width of the Venturi test section and that a small depression region is located near the throat.

Experimentally, the flow separation is evidenced by a long degassing process, with a characteristic time about $25 \mathrm{~s}$, which leads to the attachment of "tadpole" cavities that will be developed later in Sec. IV A. As we can notice in Figs. 9(a) and 9(b), first an incoming bubble grows importantly near a separation point, generally situated in the vicinity of the Venturi throat in our case, due to low pressures and detach from the wall when bubble diameter reaches the separation layer thickness. ${ }^{6}$ A part of the bubble is captured by the separation layer and is driven at the separation laminar bubble interface to the reattachment point which is usually located near the trailing edge of the Venturi $\left(X^{\star}=7.1\right)$. The bubble stabilizes to a stagnation point and, when experiencing a sufficient drag force, finally reaches back the Venturi slope to throat where it settles forming a "tadpole" attached cavity $\left(X^{\star}=Z^{\star}=0\right.$ and $\left.Y^{\star}=0.5\right)$

The bubble $\mathrm{X}$-axis velocity $U_{X}^{b}$, normalized with the inlet flow velocity $V_{i n}$, is measured with an imaging tracking technique; after image binarization, the bubble contours are detected and a center of gravity is detected. A finite-difference is then applied on the image sequence to estimate the bubble velocity. The error associated with $U_{X}^{b} / V_{\text {in }}$ is thus estimated to $10^{-3}$. This parameter is presented in Fig. $9(\mathrm{c})$ with its evolution during all the recirculating process. All the steps of this process are highlighted in this figure with first the bubble capture and deceleration into the flow separation, then its long-time stagnation at $X^{\star}=7.1$, and finally the Venturi divergent slope ascent, characterized by negative velocities, along the lateral wall $\left(Y^{\star}=0.5\right)$. 

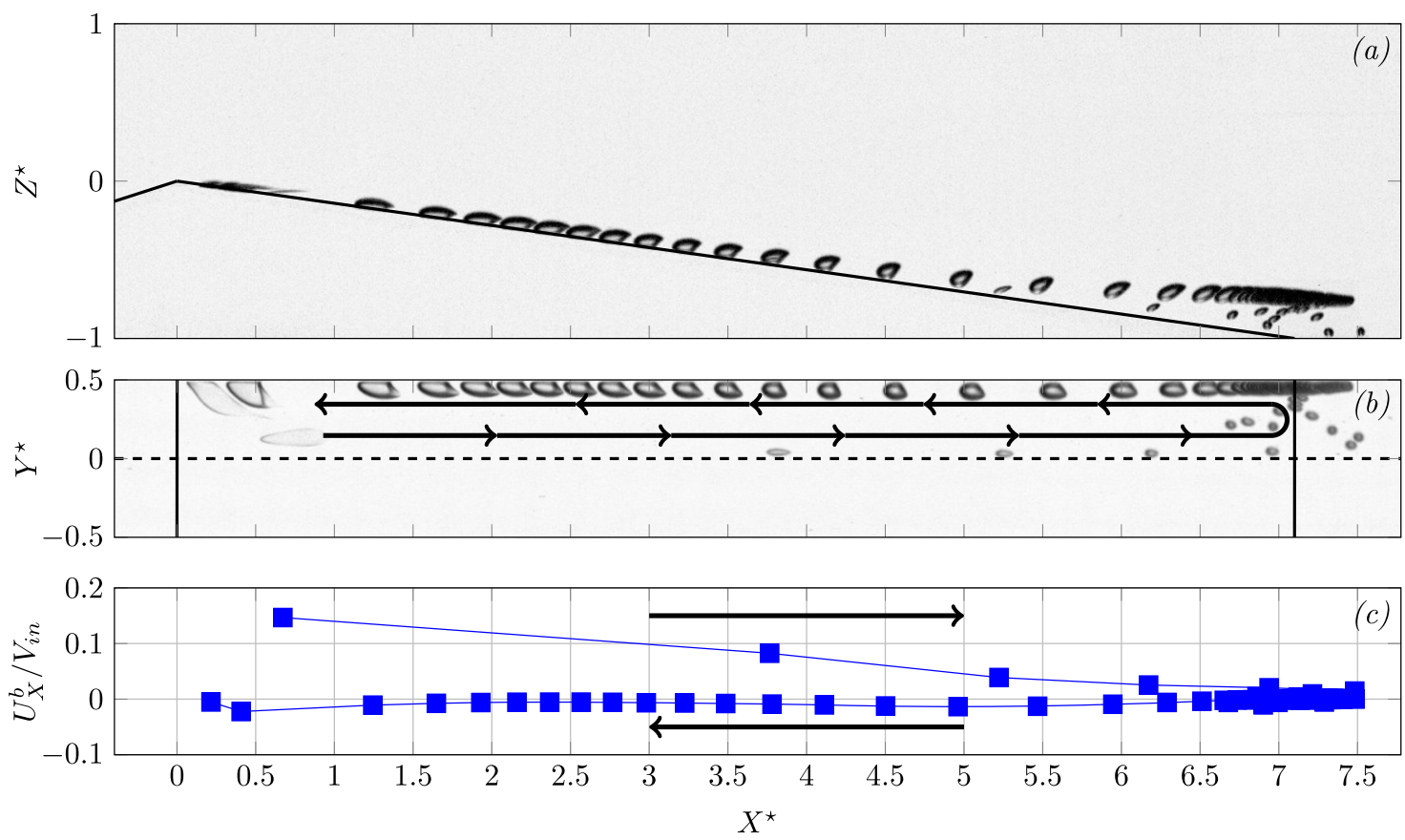

FIG. 9. Highlighting of laminar flow separation with bubble recirculation recorded at $R e \simeq 652$ and $\sigma \simeq 17.9$ in $47 \mathrm{~V} 100$ silicone oil. Side (a) and top (b) view superpositions captured during $25 \mathrm{~s}$ within a time step $\Delta t=100 \mathrm{~ms}$. (c) Evolution of the $X$-axis bubble traverse speed $U_{X}^{b}$ normalized with the inlet flow velocity $V_{\text {in }}$ and presenting an associated error about $\pm 10^{-3}$, as a function of $X^{\star}$. Black arrows indicate the direction of the recirculating bubble. Reprinted with permission from K. Croci, "Étude expérimentale des écoulements multiphasiques dans une couche limite laminaire decollée," Ph.D. thesis, Arts et Métiers Paris, France, 2018.

The recirculating process is sketched in Fig. 10 with all the different steps previously described. Consecutively to several bubble recirculations, two "tadpole" cavities attach to the Venturi throat along each lateral wall $\left(X^{\star}=Z^{\star}=0\right.$ and $\left.Y^{\star}= \pm 0.5\right)$. We assume that this recirculating phenomenon is enabled by the flow separation development on the test section width which permits the capture of an incoming bubble.

Cavity attachments in laminar separations are first investigated in Sec. IV within $47 \mathrm{~V} 100$ viscous oil, for a Reynolds number gap $R e \in[346 ; 1201]$, for which the flows are considered as steady. The flow unsteadiness, occurring at higher Reynolds numbers, is then developed in Sec. V using 47 V 50 oil until $R e=2188$.

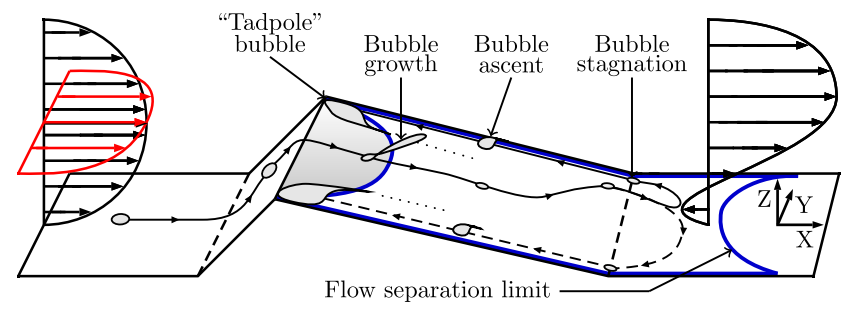

FIG. 10. Sketch of the bubble recirculation with the capture of an air bubble by the separation layer causing the attachment of a "tadpole" cavity. Reprinted with permission from K. Croci, "Étude expérimentale des écoulements multiphasiques dans une couche limite laminaire decollée," Ph.D. thesis, Arts et Métiers Paris, France, 2018.

\section{ATTACHED CAVITATION INTO LAMINAR FLOWS}

The laminar boundary layer separation presents positive effects for attached cavitation inception. A typical view of separated laminar flow, considered as steady, is depicted in Fig. 11 with the main multiphase dynamics encountered: namely, the air bubble recirculation $\left(X^{\star} \simeq 5, Y^{\star} \simeq 0.1\right.$, and $\left.Z^{\star} \simeq-0.4\right)$ leading to attached "tadpole" cavities $\left(X^{\star} \simeq 0.1, Y^{\star} \simeq \pm 0.5\right.$, and $\left.Z^{\star} \simeq 0\right)$, the attached "band" cavity $\left(X^{\star} \in[0.1 ; 0.5], Y^{\star}=Z^{\star} \simeq 0\right)$ and the secondary "tadpole" bubbles $\left(X^{\star} \in[1.5 ; 3.5], Y^{\star} \pm 0.5\right.$, and $\left.Z^{\star} \simeq 0\right)$, which will be developed in Secs. IV A-IV C.

In our study, these multiphase dynamics, which are associated with the different symbols illustrated in Fig. 11, are investigated with $47 \mathrm{~V} 100$ silicone oil as a function of Reynolds and cavitation numbers. It results in the emergence map of the different multiphase dynamics, depending on these two flow parameters, which is presented in Fig. 12.

The attached "band" cavity inception in the low pressure region near the Venturi throat, occurring for cavitation numbers $\sigma \simeq 7$, appears to follow quite nicely the numerical predictions for cavitation inception in flows supposed laminar and steady (Fig. 8). As a result, we can assume that, for the low Reynolds numbers encountered in our $47 \mathrm{~V} 100$ oil flows ( $R e \leq 1202)$, the laminar flows can be considered as quasisteady.

The secondary "tadpole" bubbles, which emerge in secondary boundary layer separations along the test section upper wall $\left(Z^{\star}=1\right)$, appears to follow a similar evolution than the "band" cavity with a cavitation number offset about -1 . This offset, which 

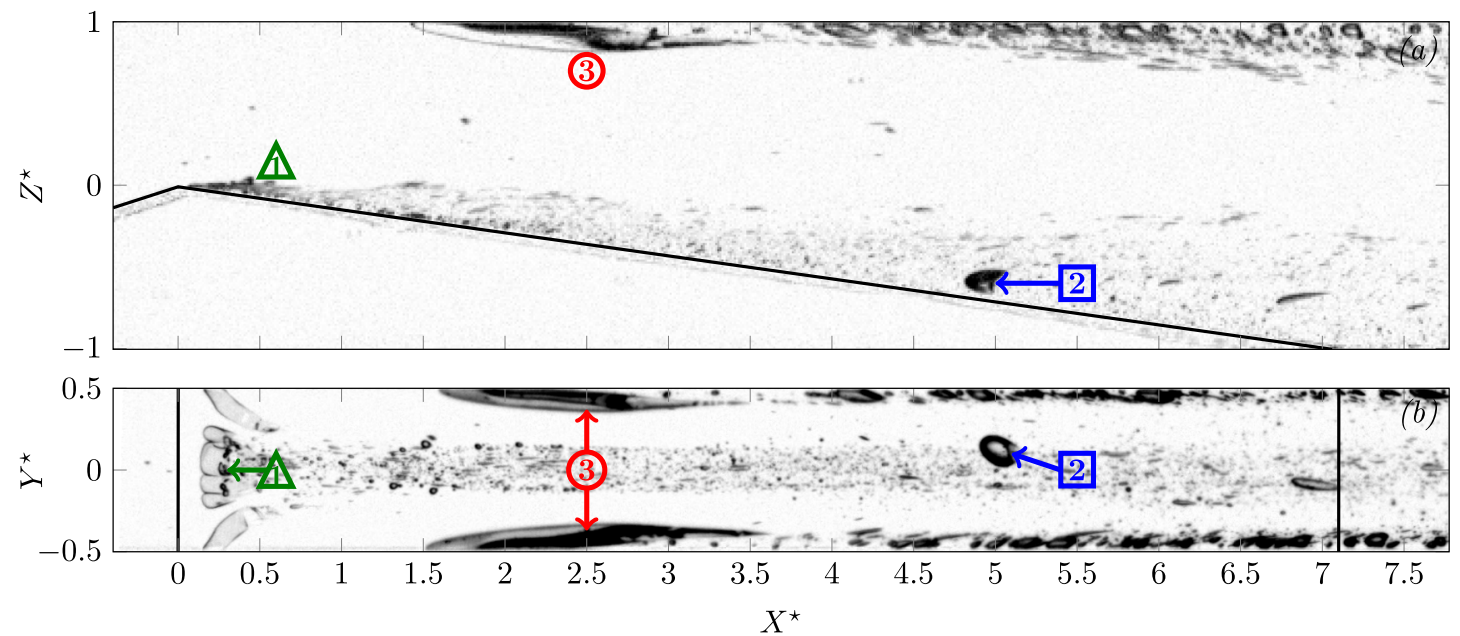

FIG. 11. Side (a) and top (b) views of a laminar multiphasic flow captured at $R e=1201$ and $\sigma=5.87$ with $47 \mathrm{~V} 100$ silicon oil. The flow is from left to right. The two black lines positioned at $X^{\star}=0$ and $X^{\star}=7.1$ indicate the throat and the end of the Venturi slope, respectively. The different symbols green open triangle, blue open square, and red open circle, numbered from 1 to 3 , represent a "band" cavity, a recirculating air bubble, and secondary "tadpole" bubbles, respectively. Reprinted with permission from $\mathrm{K}$. Croci, "Étude expérimentale des écoulements multiphasiques dans une couche limite laminaire decollée," Ph.D. thesis, Arts et Métiers Paris, France, 2018.

might be due to a dependence of secondary flow separation positions on attached "band" cavity size, will be fully developed in Sec. IV C. Finally, the attachment of "tadpole" cavities, introduced in a previous work ${ }^{29}$ with similar flow conditions, represents an interesting phenomenon which can combine both degassing and cavitation. These particular cavities can result from degassing processes, possibly occurring for a large pressure

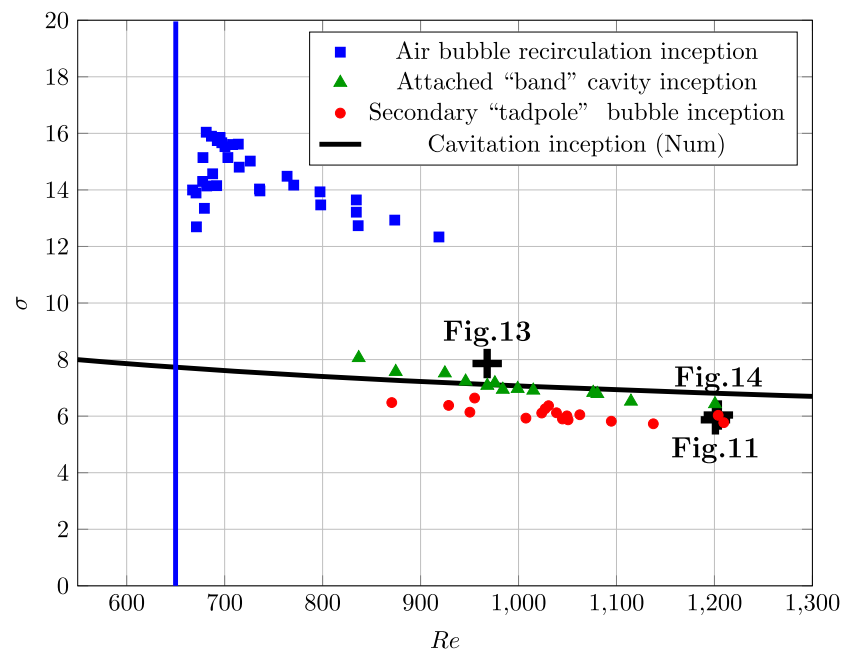

FIG. 12. Emergence map of multiphase features in silicon oil $47 \mathrm{~V} 100$. The blue line indicates the critical Reynolds number $R e_{c}^{i} \simeq 650$ from which bubbles can emerge in the separation layer and form attached cavities. Black crosses indicate the flow conditions corresponding to the different flow visualizations depicted in Sec. IV. Reprinted with permission from K. Croci, "Étude expérimentale des écoulements multiphasiques dans une couche limite laminaire decollée," Ph.D. thesis, Arts et Métiers Paris, France, 2018. array $\left(P_{v}<P_{\text {in }}<1\right.$ bar), with air bubble recirculation into the main laminar flow separation. As noticed in Croci et al. ${ }^{29}$ work, a critical Reynolds number $R e_{c}^{i} \simeq 650$ from which air bubble recirculation can occur is observed in Fig. 12. This critical number can be explained with the numerical outcomes presented in Sec. III $\mathrm{A}$ in addition to the degassing process depicted in Sec. III B.

\section{A. Attached "tadpole" cavities}

The "tadpole" cavities usually attach near the Venturi throat along the two test section wall-sides $\left(X^{\star}=Z^{\star} \simeq 0, Y^{\star}= \pm 0.5\right)$. They result from the interaction of a gas bubble with a separated flow and can be generated both with degassing or cavitation. With degassing, the attachment of these cavities follows a long process, with a characteristic time about $30 \mathrm{~s}$, which has been previously developed in Sec. III B. As previously said, this process can only occur above a critical Reynolds number $R e_{c}^{i} \simeq 650$ which correspond to the development of the flow separation all along the Venturi width observed numerically in Sec. III. This result is coherent with the degassing process, sketched in Fig. 10, with the bubble growth which occurs at the middle of the test section $\left(X^{\star} \simeq 0\right)$ and the capture of a part of this bubble by the separated flow which would be only possible if the separated flow reach this central position. As depicted in Fig. 13, the recirculating process, illustrated with the two bubbles located at $2<X^{\star}<2.5$, remains in the flow even after the attachment of "tadpole" cavities $\left(X^{\star} \simeq 0.2, Y^{\star}= \pm 0.5\right)$ inducing a bubbly recirculation regime. ${ }^{2}$

Once the "tadpole" cavities (filled with air in that case) attach to the wall, they can remain in the flow despite increasing the pressure or lowering the flow velocity whereas, in the meantime, bubble recirculation disappears. An important hysteresis between inception and desinence of "tadpole" cavities can then be 


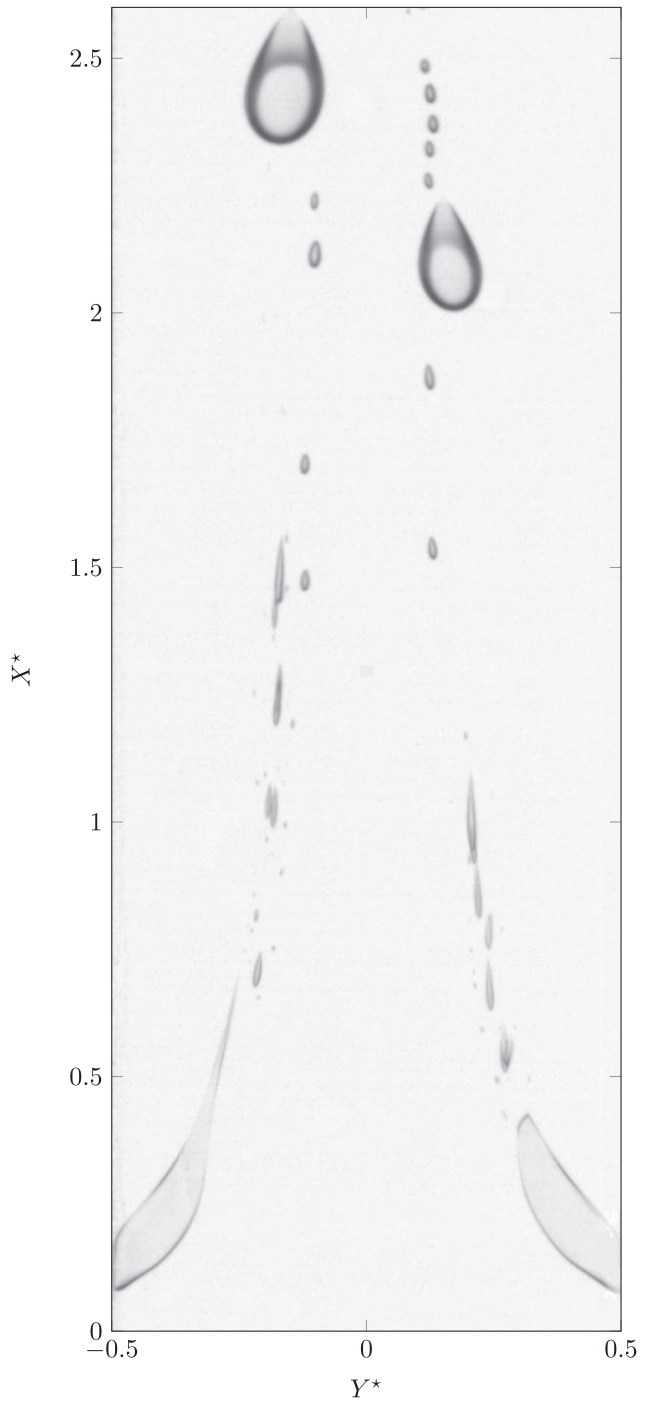

FIG. 13. Attached "tadpole" cavities top view captured at $R e=968$ and $\sigma=7.86$. Two recirculating bubbles $\left(2>X^{\star}>2.5\right)$ reach back the Venturi throat. Reprinted with permission from K. Croci, "Étude expérimentale des écoulements multiphasiques dans une couche limite laminaire decollée," Ph.D. thesis, Arts et Métiers Paris, France, 2018

observed. ${ }^{29}$ The "tadpole" cavities desinence can be generated in two ways:

- Increasing the pressure in the vicinity of the cavities above the saturating liquid pressure, which is approximately the atmospheric pressure in our experiments. The liquid becomes undersaturated and the driven diffusion ${ }^{32}$ will progressively generate the dissolution of the air contained into the cavities in the liquid.

- Decreasing the Reynolds number under a critical value, estimated in a previous work ${ }^{29}$ with the same $47 \mathrm{~V} 100$ oil to $R e_{c}^{d} \simeq 350$. This critical Reynolds number can be associated with the disappearance of laminar separations, necessary to cavity attachments, estimated in numerical computations (Sec. III) to $R e \simeq 350$.

The "tadpole" cavities, initially composed of air, can equally fill with vapor when exposed to pressures near the liquid vapor pressure. ${ }^{35}$ In situations where "tadpole" cavities cannot be generated with degassing, they can emerge on the wall-sides of the test section directly with cavitation due to unsteadiness into flows presenting higher Reynolds numbers. In these types of unsteady flows, detailed in Sec. V, an attached "band" cavity can obstruct the incoming flow and possibly generates a standing, or a "horseshoe" vortex. ${ }^{36}$ For low pressure conditions, this vortex partially vaporizes in a flow separation, generating two attached "tadpole" cavities. As a result, "tadpole" cavities can emerge at low pressures about the vapor pressure and only disappear at the high saturating liquid pressure generating an important hysteresis. A very similar phenomenon has been observed in the work of Amini et al. ${ }^{37}$ about the tip vortex cavitation on an elliptical hydrofoil.

\section{B. Attached "band" cavity}

The attached "band" cavities, illustrated in Fig. 14, are characterized by their "finger" shapes with classical "divots" structures which are due to the laminar boundary layer local perturbations interacting with the cavity. ${ }^{38}$ The "band" cavities emerge from the Venturi throat center $\left(X^{\star}=Y^{\star}=Z^{\star} \simeq 0\right)$ and develop progressively along the test section width forming more and more "divots."

They appear to be mainly composed of vapor, as evidenced by the following:

- The regular condensation of these structures observed experimentally.

- The correlation between the experimental emergence of a "band" cavity near the Venturi throat and the pressures which are estimated numerically in this region, accordingly with the corresponding flow conditions, below the vapor pressure $P_{v} \simeq 1 \mathrm{~Pa}$.

Consequently, the emergence of cavitation conditions in a continuous form provides enough vapor to stabilize the cavity despite the high velocities encountered in that flow region. The attached "band" cavities, which can coexist with air bubble recirculation, can constitute an important obstacle in the flow and can generate instabilities (Sec. V) and even influence secondary laminar separations.

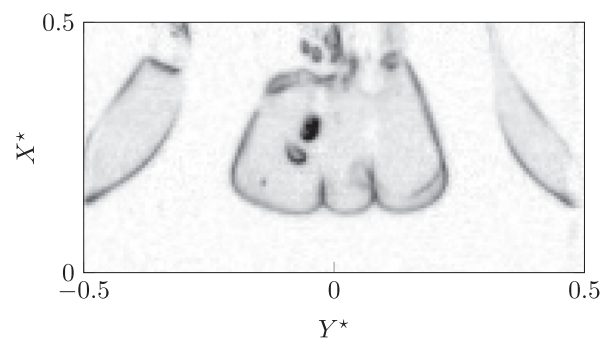

FIG. 14. Attached "band" cavity and "tadpole" cavities top view captured at $R e=1204$ and $\sigma=6.04$. Reprinted with permission from K. Croci, "Étude expérimentale des écoulements multiphasiques dans une couche limite laminaire decollée," Ph.D. thesis, Arts et Métiers Paris, France, 2018. 


\section{Secondary attached "tadpole" bubbles}

In addition to the "band" cavity and the "tadpole" cavities attachment in the main laminar flow separation, we can observe in Fig. 11 several recirculating bubbles on the upper wall of the test section $\left(X^{\star} \in[1.5 ; 3.5]\right.$ and $\left.Z^{\star}=1\right)$ along each wallside $\left(Y^{\star}= \pm 0.5\right)$. These bubbles appear to follow a recirculating dynamic, similar to that presented for "tadpole" cavities, which should indicate the presence of a secondary flow separation previously noticed numerically in Sec. III for $R e \geq 1100$. These bubbles, called secondary "tadpole" bubbles for coherency, can emerge in our experiments for much lower Reynolds numbers ( $R e \simeq 871)$. This difference can be due to the effects of attached cavities, such as the attached "band" cavity, on the flow topology whereas gas structures are not taken into account in the simulations.

Regarding the pressures encountered in this flow region and the recirculating dynamic, we can assume that these secondary "tadpole" bubbles might be mainly filled, at first, with air resulting from previous degassing features. Similar secondary flow separations have been observed by Armaly et al. ${ }^{34}$ downstream of a backward-facing step into laminar and transitioning laminar/turbulent monophase flows. They noticed that in these flows the separation point location of secondary flow separations appears to mainly depend on the reattachment point position of the main flow separation. However, in multiphase flows, the main flow separation might be importantly impacted by the development of attached cavities, such as attached "band" cavities, which can deeply change the flow topology in its wake and thus the main separated flow reattachment point position. The development of partial cavitation, as attached "band" cavities, can classically be associated with the cavitation number which characterizes how close the pressure in the liquid flow is to the vapor pressure. $^{25}$

To resume, a secondary flow separation is linked in multiphase flows to the main flow separation reattachment which might depend on attached "band" cavities' development, characterized by the flow cavitation number. Consequently, secondary "tadpole" bubbles' emergence should occur in the secondary separation consecutively to the "band" cavity inception, when the pressure is progressively decreased, and be characterized by a critical cavitation number $\sigma_{c}$. In our experiments, this inception evolution is observed in Fig. 12 and a critical cavitation number $\sigma_{c} \simeq 6.1$ is estimated.

\section{TRANSITION TO UNSTEADINESS AND VORTEX CAVITATION}

In Sec. IV, different attached cavities have been introduced in laminar separated flows considered as steady for Reynolds numbers $R e \leq 1201$. According to the numerical computations presented in Sec. III, the flow separation appears to be fully developed for higher Reynolds numbers $(R e \geq[1300 ; 1400])$ which can possibly lead to more bubbly dynamics. The attached cavities previously introduced, and more generally the bubbles observed in our experiments within their deformations, can influence the topology of the flow and possibly induce wake instabilities ${ }^{39}$ characterized by vortex cavitation at low pressures.
In the present section, we propose to extend our study to unsteady flows using a less viscous $47 \mathrm{~V} 50$ silicone oil which permits to access to a larger range of Reynolds numbers $R e \in[541 ; 2185]$.

The emergence map of the multiphase dynamic in the $47 \mathrm{~V} 50$ silicone oil is depicted in Fig. 15. As previously noticed in Sec. IV for 47 V 100 oil, degassing seems to emerge above a critical Reynolds number $R e_{c}^{i} \simeq 700$ which is consistent with the value of $R e_{c}^{i} \simeq 650$ estimated in $47 \mathrm{~V} 100$ silicone oil by Croci et al. ${ }^{29}$ Moreover, the evolution of the bubbly recirculation emergence seems to follow a similar path with the previous outcomes until $R e \simeq 1200$ where the slope of the curve completely changes and bubbly recirculation appears for higher pressure conditions. We assume that this change might be due to the instability processes which develop into the separated laminar boundary layer close to the transition to turbulence inducing pressure fluctuations. ${ }^{30}$ These fluctuations can then induce bubble growth and recirculation for higher pressure system conditions.

The inception of attached "band" cavity seems to follow a linear evolution which differs with the numerical prediction of cavitation inception, except for the lower Reynolds number point measured at $R e=1305$ and $\sigma=7.04$. We assume that for this particular point, the flow can still be considered as steady whereas for higher Reynolds numbers, unsteadiness might hasten the emergence of "band" cavity for higher pressure conditions. Finally, the secondary "tadpole" bubbles appear to still emerge in the secondary flow separation for a critical cavitation number $\sigma_{c} \simeq 6.1$.

Otherwise, transition to unsteady flows is characterized in these oil flows by vortex cavitation due to wake instabilities which can occur at relatively high Reynolds numbers. Two vortex cavitation features can be distinguished depending on the continuity of vortex vaporization:

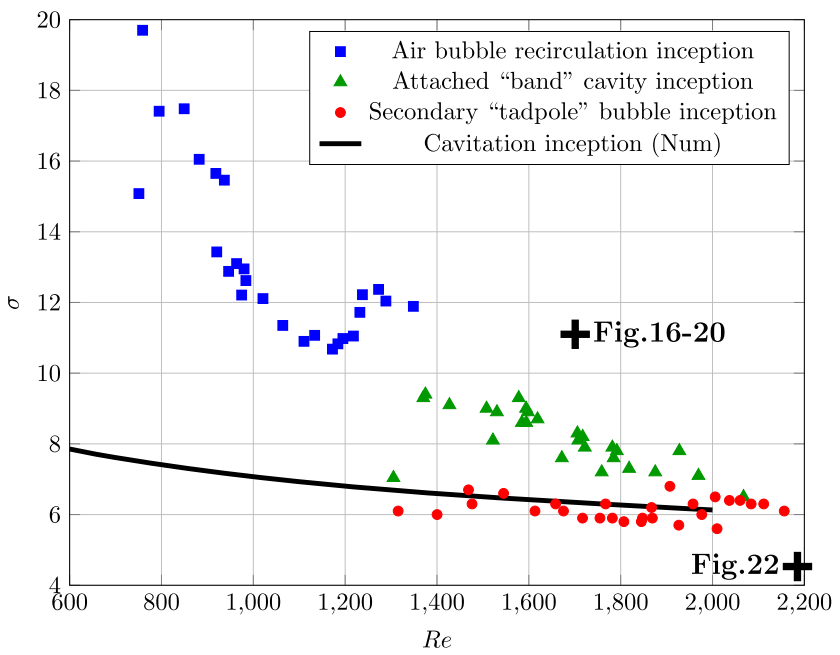

FIG. 15. Emergence map of multiphase features in $47 \mathrm{~V} 50$ silicone oil. Black crosses indicate the flow conditions corresponding to the different flow visualizations depicted in Sec. V. Reprinted with permission from K. Croci, "Étude expérimentale des écoulements multiphasiques dans une couche limite laminaire decollée," Ph.D. thesis, Arts et Métiers Paris, France, 2018. 


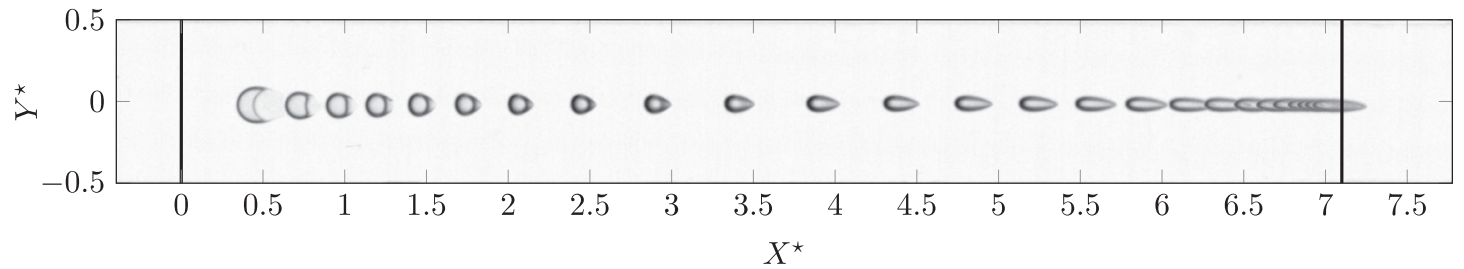

FIG. 16. Air bubble reaching back the Venturi slope to the throat at $R e=1701$ and $\sigma=11.1 \mathrm{in} 47 \mathrm{~V} 50$ oil. The sequence is captured between a time $t_{0}=0$ ms (right) and $t_{1}=240 \mathrm{~ms}$ (left) with a sampling period $\Delta t=10 \mathrm{~ms}$. The flow is from left to right. The two black lines positioned at $X^{\star}=0$ and $X_{\star}=7.1$ indicate the throat and the end of the Venturi slope, respectively. Reprinted with permission from K. Croci, "Étude expérimentale des écoulements multiphasiques dans une couche limite laminaire decollée," Ph.D. thesis, Arts et Métiers Paris, France, 2018.

- "Patch" cavitation which occur transiently in the wake of recirculating air bubbles or "band" cavities which interact with a recirculating bubble.

- The very frequent vaporization of vortices in the transitioning wake of a developed attached "band" cavity. We refer to this phenomenon as continuous vortex cavitation.

Both phenomena, inducing very quick vaporization and condensation of cavitating vortical structures, might possibly generate shock waves ${ }^{40}$ into the test section with potential erosions ${ }^{41}$ and interactions with attached cavities. To illustrate the "patch" cavitation and the continuous vortex cavitation, we focus on two $47 \mathrm{~V}$ 50 silicone oil flows presenting Reynolds numbers over 1400 and considered nearly unsteady.

\section{A. Intermittent "patch" cavitation}

$\mathrm{Li}$ and Ceccio ${ }^{13}$ observed the inception of attached "patch" cavitation in a turbulent flow due to the interaction of an incoming traveling bubble with the laminar boundary layer on a hydrofoil. The patch cavity is generated in an unstable laminar boundary layer with first the transition to turbulence of two regions from the spanwise edges of the bubble. These regions, presenting low pressure spots, are able to generate liquid vaporization with so-called cavitating "tails" which develop in the wake of the bubble to attached patches as the bubble travels. The bubble finally continues to travel on the flow direction whereas the patch cavity, detached from the bubble, remains in the flow.

\section{Experimental highlights}

In our study, a similar patch cavitation dynamic is first observed for $R e \simeq 1400$, within the frequent recirculation of air bubbles into the separated laminar flow to the Venturi throat. This phenomenon, such as those observed by $\mathrm{Li}$ and Ceccio ${ }^{13}$ into a laminar boundary layer, might indicate the early stages of transitioning mechanisms to turbulence of the viscous laminar flow. The different steps of this patch cavitation development are depicted for $R e=1701$ and $\sigma=11.1$ in Figs. 16-20.

At $R e=1701$ and $\sigma=11.1$, the flow all around the Venturi geometry remains laminar and only degassing, with air recirculating bubbles, can be noticed. An air bubble is captured by the separation layer, stabilizes to the Venturi trailing edge $\left(X^{\star} \simeq 7.1\right)$, and finally reaches back the Venturi slope when the bubble is sufficiently large to be carried by the recirculating flow. All this process lasts about $30 \mathrm{~s}$. Figure 16 illustrates the ascent of the recirculating bubble, which lasts $240 \mathrm{~ms}$, starting from a time $t_{0}$ corresponding to the moment when the bubble begins the ascent. When approaching the Venturi throat, the bubble, which presents an elongated shape in a first time, slows down and became more spherical to finally
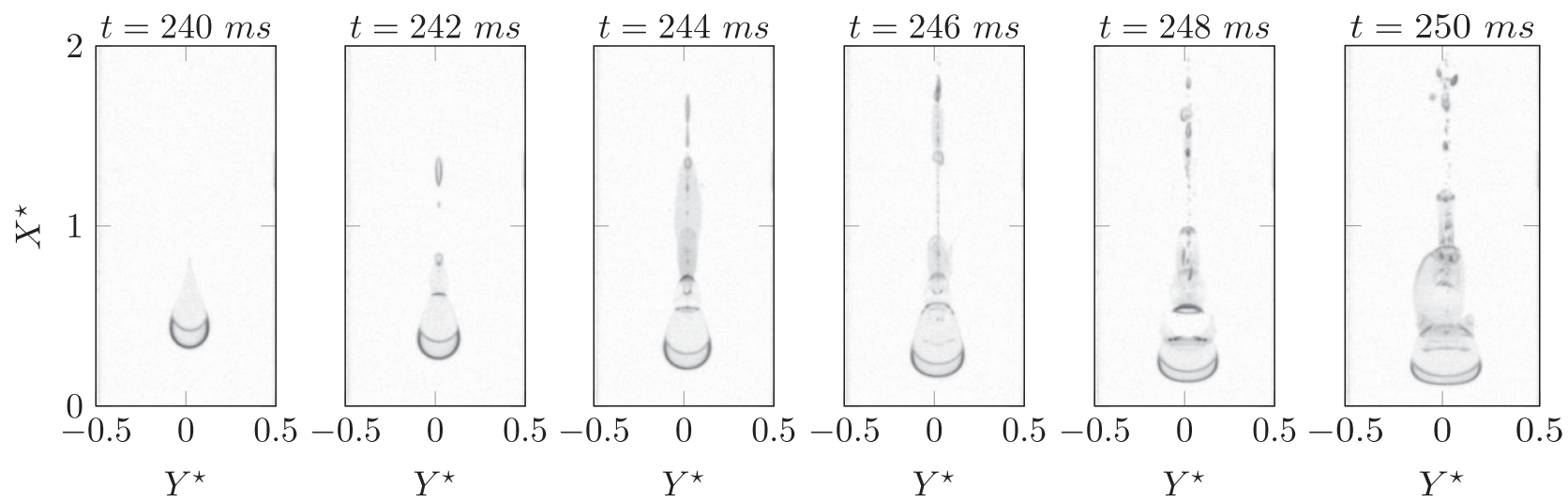

FIG. 17. Bubble deformation in the vicinity of the Venturi throat $\left(X^{*}=0\right)$ at $R e=1701$ and $\sigma=11.1$ in $47 \mathrm{~V} 50$ oil. The sequence is depicted from left to right for a time range $242<t<250 \mathrm{~ms}$ within a time step $\Delta t=2 \mathrm{~ms}$. The incoming flow go from bottom to top. Reprinted with permission from $\mathrm{K}$. Croci, "Étude expérimentale des écoulements multiphasiques dans une couche limite laminaire decollée," Ph.D. thesis, Arts et Métiers Paris, France, 2018. 

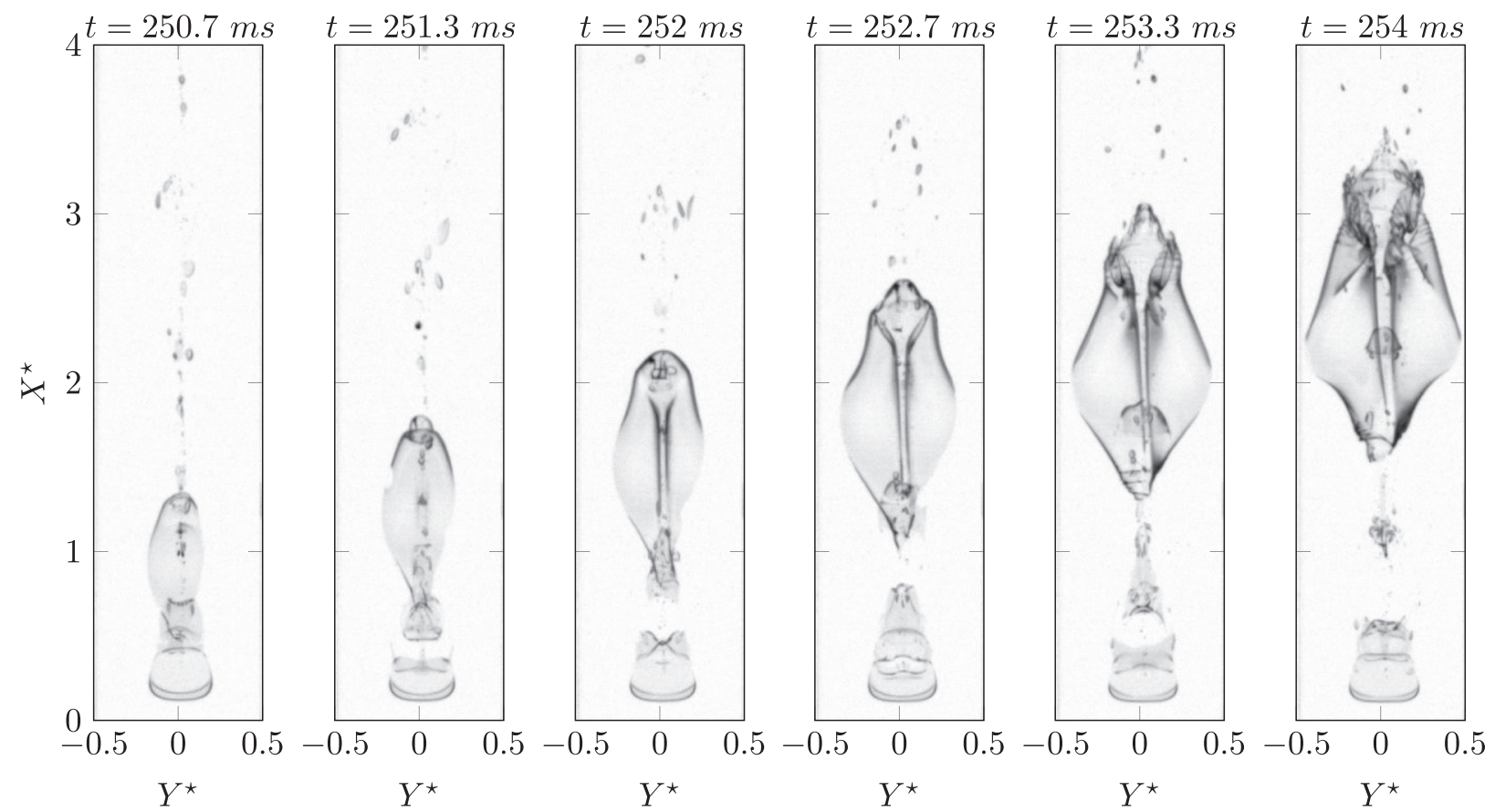

FIG. 18. Vaporization of a vortical structure in the wake of the bubble captured at $R e=1701$ and $\sigma=11.1$ in $47 \mathrm{~V} 50$ oil. The sequence is depicted from left to right for a time range $251.3<t<254 \mathrm{~ms}$ within a time step $\Delta t=2 / 3 \mathrm{~ms}$. Reprinted with permission from $\mathrm{K}$. Croci, "Étude expérimentale des écoulements multiphasiques dans une couche limite laminaire decollée," Ph.D. thesis, Arts et Métiers Paris, France, 2018.

experience a rapid deformation near the throat, within a few milliseconds $(242<t<250 \mathrm{~ms})$, called a "hemispherical cap" by $\mathrm{Li}$ and Ceccio. ${ }^{13}$ According to their study, this bubble deformation, illustrated in our experiments in Fig. 17, occurs when the distance between the bubble and the wall surface becomes similar to the boundary layer thickness. In our viscous flow, we can notice that, during this deformation, microbubble air shedding takes place in the rear of the bubble in addition to oil vaporization (located at $Y^{\star}=0$ and for $X^{\star}>0.5$ in Fig. 17). We can then assume that the air bubble, acting as an obstacle in the flow in the vicinity of the throat, can cause a pressure drop in its wake favorable for bubble vapor filling and can induce instabilities in the laminar boundary layer which might lead to the transition to turbulence.

In our example, these instabilities are revealed by the vaporization of a vortical structure in a small time range $251.3<t$ $<254 \mathrm{~ms}$ depicted in Fig. 18. The side view of the structure captured at $t=253.3 \mathrm{~ms}$ is depicted in Fig. 19. Besides, similar structure can be observed in the wake of a rising bubble experiencing the zigzagging regime at the transition to path instability. ${ }^{42}$ This well-developed cavitating structure condenses rapidly at higher pressure conditions,

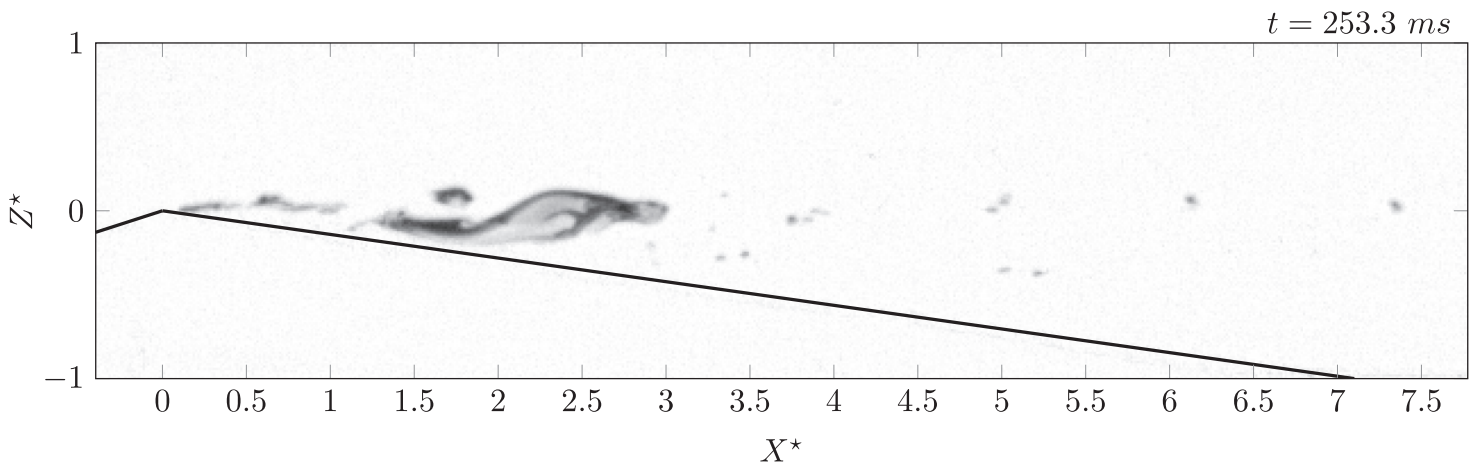

FIG. 19. Side view of the cavitating vortical structure observed at $t=253.3$ for $R e=1701$ and $\sigma=11.1$ in $47 \mathrm{~V} 50$ oil. The flow is from left to right. The corresponding top view is visible in Fig. 18. Reprinted with permission from K. Croci, "Étude expérimentale des écoulements multiphasiques dans une couche limite laminaire decollée," Ph.D. thesis, Arts et Métiers Paris, France, 2018. 

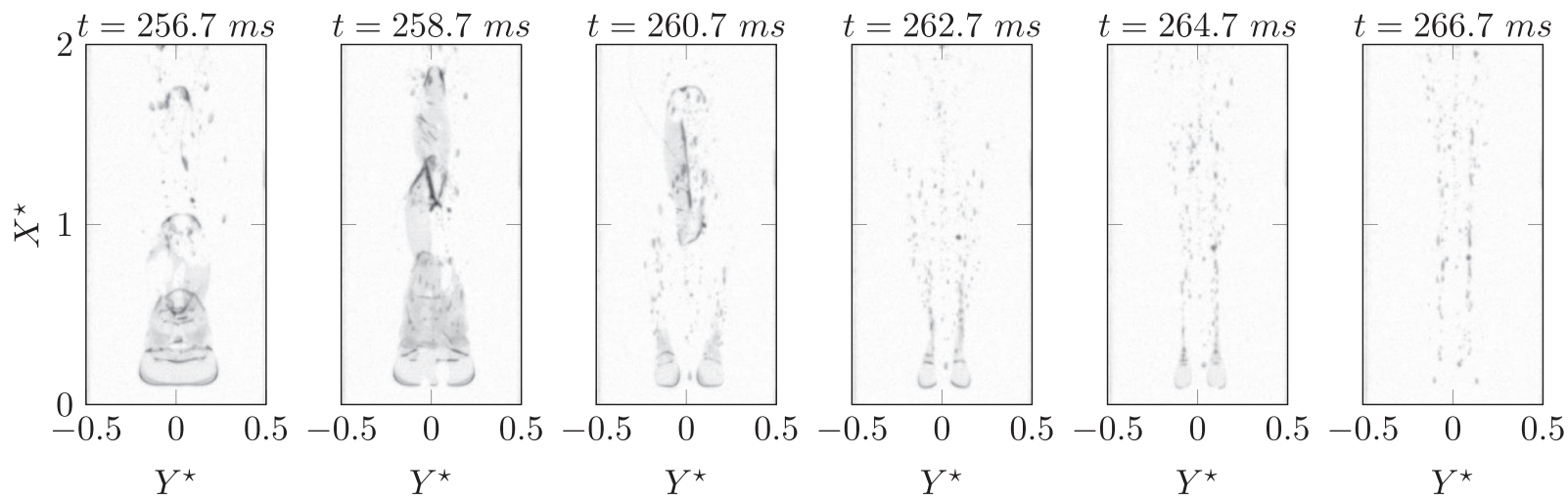

FIG. 20. Condensation of the bubble, filled with vapor, captured at $R e=1701$ and $\sigma=11.1$ in $47 \mathrm{~V} 50$ oil. The sequence is depicted from left to right for a time range $258.7<t<266.7 \mathrm{~ms}$ within a time step $\Delta t=2 \mathrm{~ms}$. Reprinted with permission from K. Croci, "Étude expérimentale des écoulements multiphasiques dans une couche limite laminaire decollée," Ph.D. thesis, Arts et Métiers Paris, France, 2018.

and consecutively, the cap shape bubble established near the Venturi throat vanishes progressively between $t=258.7 \mathrm{~ms}$ and $t=266.7 \mathrm{~ms}$ as shown in Fig. 20. The rapid condensation of the vaporized vortical structure, which completely disappears in the flow after $20 \mathrm{~ms}$, might lead to the generation of pressure waves propagating throughout the test section. As the pressure increases in the bubble, the center part of the bubble, which might be mainly composed of vapor, condenses leading to two separated bubbles. These two bubbles finally vanish with the combination of air shedding and oil vapor condensation. All the patch cavitation process depicted from Figs. 16-20 is sketched into Fig. 21.

To conclude, the phenomenon described differs a little bit from the patch cavitation described by $\mathrm{Li}$ and Ceccio, ${ }^{13}$ who observed the patch cavity emergence due to a traveling bubble passing through
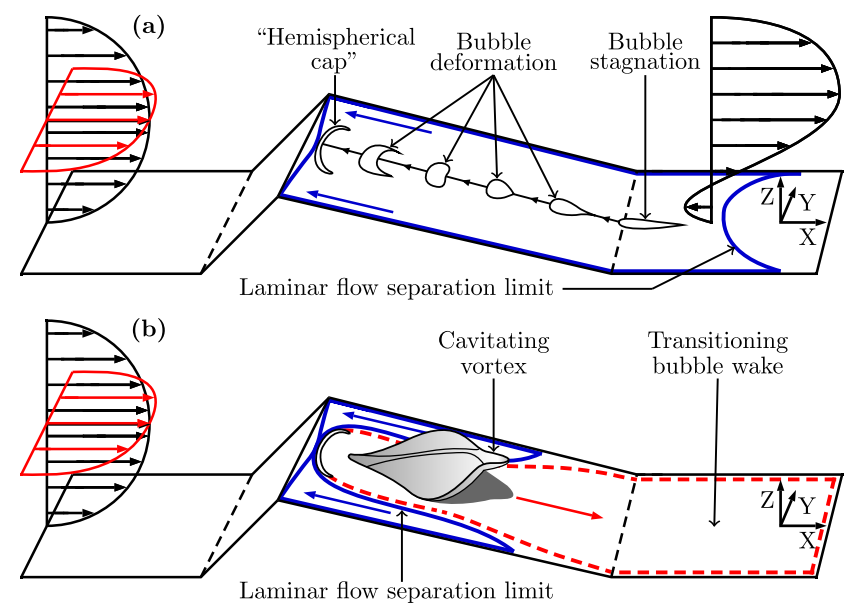

FIG. 21. Sketch of the patch cavitation process with the bubble ascent (a) with the deformation to "hemispherical cap" and transition to turbulence (b) in the bubble wake with a vortical structure vaporization. Reprinted with permission from K. Croci, "Étude expérimentale des écoulements multiphasiques dans une couche limite laminaire decollée," Ph.D. thesis, Arts et Métiers Paris, France, 2018. a laminar boundary layer into a turbulent flow and not a recirculating bubble in a laminar separated flow as in our experiments. However, the similarities evidenced, with the cap deformation of the bubble or the local transitions to turbulence, allow us to associate the phenomenon previously described to patch cavitation. This patch cavitation description can also highlight the progressive filling of the initial air bubble with oil vapor which is usually complicated to visualize.

\section{Instability transition analysis}

In the process of "patch" cavitation, we just presented, a regime transition appears to occur after an important deformation of the bubble which can be considered as an obstacle in the oil stream. We propose to compare the transition conditions encountered in our experiment in the wake of the deformed bubble to the literature about rising bubble wake transition. Ormière and Provansal ${ }^{43}$ measured the transition to turbulence in the wake of a sphere with a critical Reynolds number $\operatorname{Re}_{c}=U_{t} d / v \simeq 280$ with $d$ the sphere diameter. However, real bubbles are usually not spherical and present a deformation. In the wake of a rising bubble, with an aspect ratio about $\gamma \simeq 1.85$, Duineveld ${ }^{44}$ found a critical bubble Reynolds number $R e_{c}^{b}=U_{t} d_{e q} / v \simeq 660$, with $d_{e q}$ the equivalent spherical bubble diameter, for the transition to unsteadiness. Magnaudet and Mougin $^{39}$ added that the transition is highly related to a critical bubble aspect ratio $\gamma_{c} \simeq 2.21$ for which the wake instabilities are able to occur.

In our experiment, we observe the emergence of a cavitating structure, which might result from a vortex vaporization, after a very quick bubble deformation within a few milliseconds. When the vortical structure begin to vaporize at $t=250 \mathrm{~ms}$ (Fig. 17), the bubble present a width $w_{b} \simeq 3.6 \pm 0.1 \mathrm{~mm}$ and an aspect ratio $\gamma_{b} \simeq 7.2 \pm 0.3$. Assimilating the bubble width in our experiment to the equivalent rising bubble diameter calculated in Duineveld's ${ }^{44}$ work, we obtain a similar critical bubble Reynolds number about $R e_{c}^{b} \simeq 616 \pm 5 \%$ to characterize the emergence of wake instabilities and, in our case, vortex cavitation. Moreover, with the aspect ratio we measured being much higher than the critical aspect ratio proposed by Magnaudet and Mougin, ${ }^{39}$ we can assume that all the 


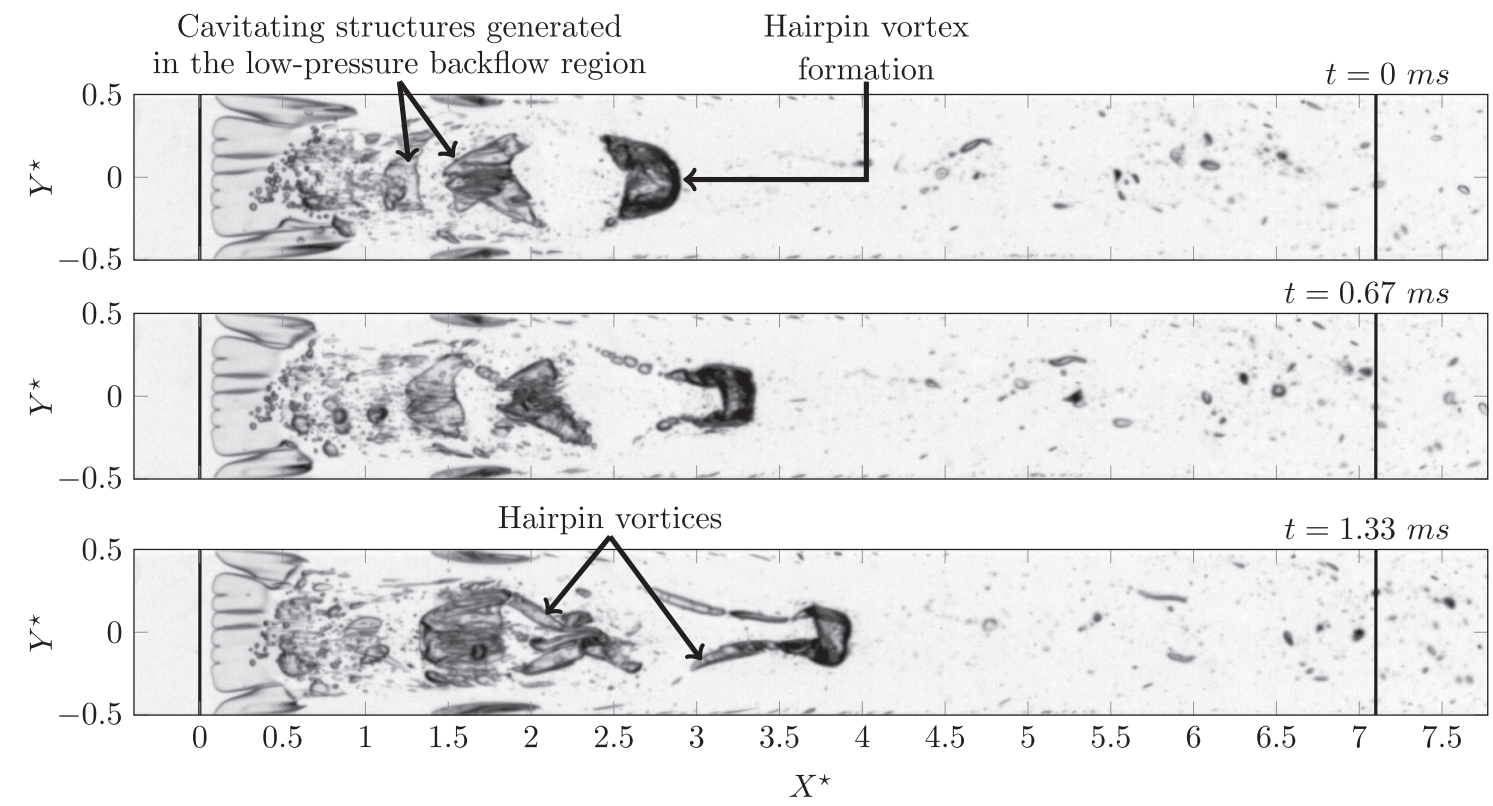

FIG. 22. Vaporization of a hairpin vortex in the wake of an attached band cavity captured at $R e=2185$ and $\sigma=4.53$ in $47 \mathrm{~V} 50$ oil. The sequence is observed from top to bottom with a period $\Delta t=2 / 3 \mathrm{~ms}$. The flow is from left to right. Reprinted with permission from K. Croci, "Étude expérimentale des écoulements multiphasiques dans une couche limite laminaire decollée," Ph.D. thesis, Arts et Métiers Paris, France, 2018.

conditions for bubble wake transition to instabilities are met. More generally, "patch" cavitation is experimentally observed in almost all the multiphase flows presenting Reynolds numbers over 1400 and attached "band" cavities not fully developed along the Venturi throat $(\sigma>4.6)$. In these conditions, the wake instabilities cannot occur continuously and "patch" cavitation would emerge intermittently depending on bubbly recirculation which brings gas to the Venturi throat.

\section{B. Continuous vortex cavitation}

A continuous flow regime transition can occur in the wake of developed attached "band" cavities for high Reynolds numbers. An example of cavitating flow in a laminar/turbulent transition regime with $R e=2185$ and $\sigma=4.53$ is depicted in Fig. 22. This transitioning flow is characterized by the frequent vaporization of vortex structures, identified as a hairpin vortex, $\left(X^{\star} \in[3 ; 4]\right.$ at $\left.t=1.33 \mathrm{~ms}\right)$ in the wake of a "band" cavity which condenses downstream with higher pressure conditions. This vortex cavitation appears to occur at highfrequency, requiring more efficient high speed cameras. The study of such dynamics, within the impact of vapor structures' collapse on the flow topology, will be focused in a future work. However, the frequency of occurrence of such phenomenon can be estimated with a method similar to the one used by Croci et al.: ${ }^{45}$ with a wavelet method applied on the top view image sequence depicted in Fig. 22. A wavelet analysis is applied on the gray level signal extracted from a square region $\left(1.8<X^{\star}<2.3 ;-0.25<Y^{\star}<0.25\right)$, located at the maximum of standard deviation of the image sequence. As a result, vapor structures, which we assimilate to vaporizing hairpin vortices, emerge with an estimated frequency $f \simeq 350 \mathrm{~Hz} \pm 4 \%$, corresponding to a Strouhal number $S t=f \times w / U_{\text {in }} \simeq 0.6$.

The separated steady laminar flows, which presented large main boundary layer separations with reattaching point usually located over the Venturi trailing edge $\left(X^{\star}>7.1\right)$, reduce importantly with

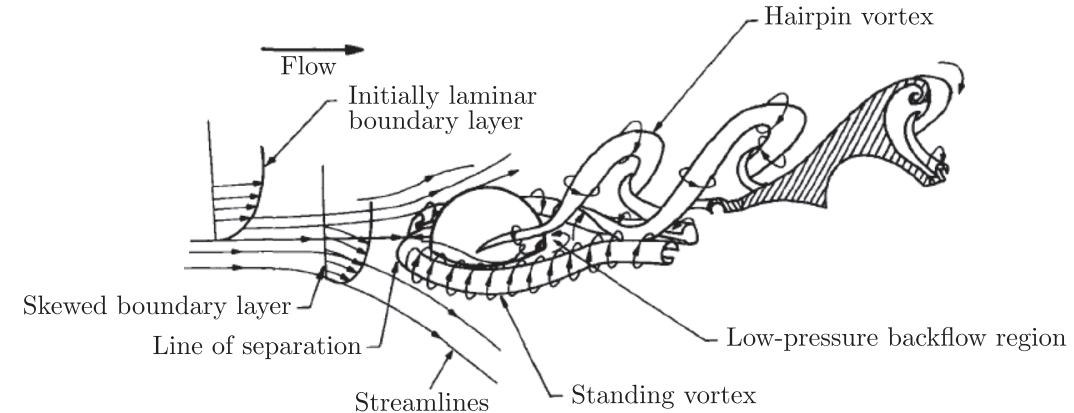

FIG. 23. Sketch of the emergence of a hairpin votex in the wake of a hemisphere protuberance. Reproduced with permission from M. S. Acarlar and C. R. Smith, "A study of hairpin vortices in a laminar boundary layer. Part 1. Hairpin vortices generated by a hemisphere protuberance," J. Fluid Mech. 175, 1-41 (1987). Copyright 1987 Cambridge University Press. 
transition to laminar/turbulent transitioning regime forming a laminar separation bubble. ${ }^{2}$ We thus estimate that the reattaching point along the Venturi slope in the transitioning flow presented in Fig. 22 is located at $X^{\star} \simeq 1$.

The vortex formation in our experiments can be compared to those observed by Acarlar and Smith ${ }^{36}$ about a hemisphere protuberance. In their experiments, as shown in Fig. 23, the protuberance induces a flow regime transition characterized by hairpin vortices which developed frequently in its wake in addition to a standing or horseshoe vortex around the protuberance. In our experiments, the protuberance can be assimilated to the attached "band" cavity and we can assume that hairpin vortices are generated similarly with, in our case, the possible vaporization of a part of the backflow region which might quite modify the flow inducing other vortex vaporizations. We also suppose that a horseshoe vortex might eventually vaporize at low pressures around a "band" cavity generating the vapor "tadpole" cavities on the flow separation located on the wall sides $\left(Y^{\star} \pm 0.5\right)$.

\section{CONCLUSIONS AND FUTURE WORKS}

In the present study, multiphase separated flows have been investigated experimentally focusing on attached cavities and their influence on the flow stability. One-phase numerical simulations have been performed additionally to illustrate the flow topology depending on the two main flow parameters: the Reynolds and the cavitation numbers.

As a result, flow separations have been highlighted both numerically and experimentally with bubbly recirculation. A separated laminar flow can experience both degassing and cavitation possibly generating different attached cavities: "band" and "tadpole" cavities in the main flow separation along the Venturi geometry and secondary "tadpole" bubbles in a secondary flow separation located on the upper wall of the test section. Both "tadpole" cavities and secondary "tadpole" bubbles appear to be mainly associated with degassing and bubble recirculation which cause their attachment into flow separations. On the other hand, the attached "band" can be associated with cavitation inception into the low pressure region induced by the Venturi geometry and located at the throat. The initial steady laminar flow can become unstable due to bubble wake instabilities for flow Reynolds numbers $R e \geq 1400$, corresponding to a critical bubble Reynolds number $\operatorname{Re}_{c}^{b} \simeq 616$. Above these values, a transition to unsteadiness can take place in the wake of developed cavities or bubbles intermittently. The vortex cavitation occurs with a frequency estimated to $f \simeq 350 \mathrm{~Hz}$ in the present study. A future work will consist in investigating the development of attached "band" cavities into degassed separated flows focusing on wake vortex cavitation, with relevant frequency analyses, until the transition to supercavitation.

Otherwise, future progress in the comprehension of multiphase viscous flows requires adapted measurement devices to control and estimate precisely bubble gas composition and dissolved gas content in the flow. Velocity measurements into the separated boundary layer might be also appreciated to validate the numerical outcomes. The development of partial cavitation at low Reynolds numbers should also be considered regarding the evolution of attached cavitation in a large range of Reynolds numbers going from 2000 to $10^{5}$, the characteristic value encountered in turbulent water flows, ${ }^{31}$ with a focus on the emergence of the classical re-entrant jet shedding mechanism in the flow.

\section{ACKNOWLEDGMENTS}

This research is part of the Ph.D. thesis realized by $\mathrm{Croci}^{46}$ about the "Experimental study of multiphase flows within a separated laminar boundary layer."

\section{REFERENCES}

${ }^{1}$ A. Dovgal, V. Kozlov, and A. Michalke, "Laminar boundary layer separation: Instability and associated phenomena," Prog. Aeronaut. Sci. 30, 61-94 (1994).

${ }^{2} \mathrm{M}$. Gaster, "The structure and behaviour of laminar separation bubbles," NPL Aero Report No. 1181, 1992, revised.

${ }^{3}$ V. H. Arakeri, "Viscous effects on the position of cavitation separation from smooth bodies," J. Fluid Mech. 68, 779-799 (1975).

${ }^{4}$ J.-P. Franc and J.-M. Michel, "Attached cavitation and the boundary layer: Experimental investigation and numerical treatment," J. Fluid Mech. 154, 63-90 (1985).

${ }^{\mathbf{5}}$ B. R. Parkin and R. W. Kermeen, "Incipient cavitation and boundary layer interaction on a streamlined body," California Institute of Technology Hydraulic Laboratory, Report No. E-35.2, 1953.

${ }^{6}$ V. H. Arakeri, "Viscous effects in inception and development of cavitation on axi-symmetric bodies," Ph.D. thesis, California Institute of Technology, USA, 1973.

${ }^{7}$ G. Kuiper, “Cavitation inception on ship propeller models," Ph.D. thesis, Delft University of Technology, The Netherlands, 1981.

${ }^{8}$ M. F. Guennoun, "Étude physique de l'apparition et du développement de la cavitation sur une aube isolée," Ph.D. thesis, EPFL Lausanne, Switzerland, 2006.

${ }^{9}$ P. Brandner, G. J. Walker, P. N. Niekamp, and B. Anderson, “An experimental investigation of cloud cavitation about a sphere," J. Fluid Mech. 656, 147-176 (2010).

${ }^{10}$ P. F. Pelz, T. Keil, and T. F. Groß, "The transition from sheet to cloud cavitation," J. Fluid Mech. 817, 439-454 (2017).

${ }^{11}$ M. Callenaere, J.-P. Franc, J.-M. Michel, and M. Riondet, "The cavitation instability induced by the development of a re-entrant jet," J. Fluid Mech. 444, 223-256 (2001).

${ }^{12}$ H. Ganesh, S. A. Mäkiharju, and S. L. Ceccio, "Bubbly shock propagation as a mechanism for sheet-to-cloud transition of partial cavities," Phys. Fluids 802, 37-78 (2016).

${ }^{13}$ C. Y. Li and S. L. Ceccio, "Interaction of single travelling bubbles with the boundary layer and attached cavitation," J. Fluid Mech. 322, 329-353 (1996).

${ }^{14}$ M. van Rijsbergen, "A review of sheet cavitation inception mechanisms," in ISROMAC2016-International Symposium on Transport Phenomena and Dynamics of Rotating Machinery, Hawaii, Honolulu, USA, 2016.

${ }^{15} \mathrm{M}$. Briançon-Marjollet, J.-P. Franc, and J.-M. Michel, "Transient bubbles interacting with an attached cavity and the boundary layer," J. Fluid Mech. 218, 355-376 (1990).

${ }^{16}$ J.-P. Franc and J.-M. Michel, Fundamentals of Cavitation (Kluwer Academic Publishers, Dordrecht, Boston, 2006).

${ }^{17}$ J. A. Venning, M. T. Khoo, B. W. Pearce, and P. A. Brandner, "Background nuclei measurements and implications for cavitation inception in hydrodynamic test facilities,” Exp. Fluids 59, 71 (2018).

${ }^{18}$ J. W. Holl and A. L. Treaster, “Cavitation hysteresis,” J. Basic Eng. 88, 199-212 (1966).

${ }^{19}$ Y. Kodama, N. Take, S. Tamiya, and H. Kato, "The effect of nuclei on the inception of bubble and sheet cavitation on axisymmetric bodies," J. Fluids Eng. 103, 557-563 (1981).

${ }^{20} \mathrm{~B}$. Li, Y. Gu, and M. Chen, "An experimental study on the cavitation of water with dissolved gases,” Exp. Fluids 58, 164 (2017).

${ }^{21}$ F. Peters and R. Honza, “A benchmark experiment on gas cavitation," Exp. Fluids 55, 1786 (2014).

${ }^{22} \mathrm{C}$. Ding and Y. Fan, "Measurement of diffusion coefficients of air in silicone oil and in hydraulic oil," Chin. J. Chem. Eng. 19, 205-211 (2011). 
${ }^{23}$ H.-A. Freudigmann, A. Dörr, U. Iben, and P. F. Pelz, "Modeling of cavitationinduced air release phenomena in micro-orifice flows," J. Fluids Eng. 139, 111301 (2017).

${ }^{24}$ T. Ishihara, M. Ouchi, T. Kobayashi, and N. Tamura, "An experimental study on cavitation in unsteady oil flow," Bull. JSME 22, 1099-1106 (1979).

${ }^{25}$ C. E. Brennen, Fundamentals of Multiphase Flows (Cambridge University Press, 2005).

${ }^{26} \mathrm{~S}$. Washio, S. Takahashi, and S. Yoshimori, "Study on cavitation starting at the point of separation on a smooth wall in hydraulic oil flow," Proc. Inst. Mech. Eng., Part C 217, 619-630 (2003).

${ }^{27}$ S. Washio, S. Takahashi, K. Uemura, T. Iwamoto, and T. Ogata, "Singular properties of flow separation as a real cause of cavitation inception," Proc. Inst. Mech. Eng., Part C 222, 667-678 (2008).

${ }^{28}$ S. Washio, S. Kikui, and S. Takahashi, "Nucleation and subsequent cavitation in a hydraulic oil poppet valve," Proc. Inst. Mech. Eng., Part C 224, 947-958 (2009).

${ }^{29}$ K. Croci, F. Ravelet, J.-C. Robinet, and A. Danlos, "Experimental study of cavitation in laminar flow," in 10th Symposium on Cavitation, Baltimore, USA, May 2018.

${ }^{30}$ J. Katz, "Cavitation phenomena within regions of flow separation," J. Fluid Mech. 140, 397-436 (1984).

${ }^{31}$ A. Danlos, F. Ravelet, O. Coutier-Delgosha, and F. Bakir, "Cavitation regime detection through proper orthogonal decomposition: Dynamics analysis of the sheet cavity on a grooved convergent divergent nozzle," Int. J. Heat Fluid Flow 47, 9-20 (2014).

${ }^{32}$ T. F. Groß and P. F. Pelz, "Diffusion-driven nucleation from surface nuclei in hydrodynamic cavitation," J. Fluid Mech. 830, 138-164 (2017).

${ }^{33}$ G. Duhar and C. Colin, "Dynamics of bubble growth and detachment in a viscous shear flow," Phys. Fluids 18, 077101 (2006).

${ }^{34}$ B. F. Armaly, F. Durst, J. C. F. Pereira, and B. Schönung, "Experimental and theoretical investigation of backward-facing step flow," J. Fluid Mech. 127, 473-496 (1983).
${ }^{35}$ T. Koivula, "On cavitation in fluid power," in Proceedings of the 1st FPNIPh.D. Symposium, Hamburg 2000 (Slovak University of Technology Press, 2000), pp. 371-382.

${ }^{36}$ M. S. Acarlar and C. R. Smith, "A study of hairpin vortices in a laminar boundary layer. Part 1 . Hairpin vortices generated by a hemisphere protuberance," J. Fluid Mech. 175, 1-41 (1987).

${ }^{37}$ A. Amini, M. Reclari, T. Sano, and M. Farhat, "Effect of gas content on tip vortex cavitation," in Symposium on Cavitation, 2018.

${ }^{38}$ A. Tassin Leger, L. P. Bernal, and S. L. Ceccio, "Examination of the flow near the leading edge of attached cavitation. Part 2. Incipient breakdown of two-dimensional and axisymmetric cavities," J. Fluid Mech. 376, 91-113 (1998).

${ }^{39}$ J. Magnaudet and G. Mougin, "Wake instability of a fixed spheroidal bubble," J. Fluid Mech. 572, 311-337 (2007).

${ }^{40}$ W. Garen, F. Hegedus, Y. Kai, S. Koch, B. Meyerer, W. Neu, and U. Teubner, "Shock wave emission during the collapse of cavitation bubbles," Shock Waves $\mathbf{2 6}$, 385-394 (2016).

${ }^{41}$ M. Dular and M. Petkovšek, "On the mechanisms of cavitation erosioncoupling high speed videos to damage patterns," Exp. Therm. Fluid Sci. 68, 359-370 (2015).

${ }^{42}$ J. C. Cano-Lozano, C. Martinez-Bazan, J. Magnaudet, and J. Tchoufag, "Paths and wakes of deformable nearly spheroidal rising bubbles close to the transition to path instability," Phys. Rev. Fluids 1, 053604 (2016).

${ }^{43} \mathrm{D}$. Ormière and M. Provansal, "Transition to turbulence in the wake of a sphere," Phys. Rev. Lett. 83, 80 (1999).

${ }^{44} \mathrm{P}$. C. Duineveld, "The rise velocity and shape of bubbles in pure water at high Reynolds number," J. Fluid Mech. 292, 325-332 (1995).

${ }^{45}$ K. Croci, P. Tomov, F. Ravelet, A. Danlos, S. Khelladi, and J.-C. Robinet, "Investigation of two mechanisms governing cloud cavitation shedding: Experimental study and numerical highlight," in ASME 2018 International Mechanical Engineering Congress and Exposition, Fluids Engineering (ASME, 2016), Vol. 7.

${ }^{46} \mathrm{~K}$. Croci, "Étude expérimentale des écoulements multiphasiques dans une couche limite laminaire décollée," Ph.D. thesis, Arts et Métiers Paris, France, 2018. 